\title{
Impacts des barrages sur les caractéristiques des débits moyens annuels en fonction du mode de gestion et de la taille des bassins versants au Québec
}

\section{The effects of dams on mean anual flow characteristics according to management mode and basin drainage area in Québec}

\author{
Ali A. Assani, Francis Lajoie et Charles Laliberté
}

Volume 20, numéro 1, 2007

URI : https://id.erudit.org/iderudit/015741ar

DOI : https://doi.org/10.7202/015741ar

Aller au sommaire du numéro

\section{Éditeur(s)}

Université du Québec - INRS-Eau, Terre et Environnement (INRS-ETE)

\section{ISSN}

1718-8598 (numérique)

Découvrir la revue

Citer cet article

Assani, A. A., Lajoie, F. \& Laliberté, C. (2007). Impacts des barrages sur les caractéristiques des débits moyens annuels en fonction du mode de gestion et de la taille des bassins versants au Québec. Revue des sciences de l'eau / Journal of Water Science, 20(1), 127-146. https://doi.org/10.7202/015741ar

\section{Résumé de l'article}

Nous avons comparé les impacts des barrages sur les caractéristiques (volume d'écoulement-fréquence, variabilité interannuelle et forme de courbe de distribution) des débits moyens annuels dans les trois régimes régularisés observés (inversion, homogénéisation et type naturel) au Québec. Nous avons appliqué la méthode de proportionnalité qui consiste à comparer les caractéristiques des débits des rivières naturelles et celles des rivières régularisées en fonction de la taille des bassins versants. En ce qui concerne le volume d'écoulement-fréquence et sa variabilité interannuelle, le changement a été observé seulement en régime d'inversion. Il se traduit par une baisse des débits moyens annuels durant les années hydrologiques sèches et une variabilité interannuelle relativement forte par rapport aux rivières naturelles. Ces changements sont attribués principalement au mode de gestion des réservoirs car on lâche moins d'eau durant ces années hydrologiques sèches. Enfin, les changements des coefficients d'asymétrie et d'aplatissement ont été observés surtout en régime d'homogénéisation. Cette étude démontre que les barrages peuvent modifier toutes les caractéristiques des débits moyens annuels contrairement à l'opinion couramment admise. 


\title{
IMPACTS DES BARRAGES SUR LES CARACTÉRISTIQUES DES DÉBITS MOYENS ANNUELS EN FONCTION DU MODE DE GESTION ET DE LA TAILLE DES BASSINS VERSANTS AU QUÉBEC
}

\author{
The effects of dams on mean anual flow characteristics according to management mode and basin drainage area in \\ Québec
}

\begin{abstract}
Ali A. Assani ${ }^{1 *}$, Francis Lajoie ${ }^{1}$ et Charles Lalibertét
${ }^{1}$ Laboratoire d'hydro-climatologie et de géomorphologie fuviale, Section de géographie, Pavillon Léon-Provencher, Université du Québec à TroisRivières, 3351, boulevard Des Forges, Trois-Rivières (Québec), Canada G9A 5 H7
\end{abstract}

Reçu le 7 novembre 2005, accepté le 3 août 2006

\section{RÉSUMÉ}

Nous avons comparé les impacts des barrages sur les caractéristiques (volume d'écoulement-fréquence, variabilité interannuelle et forme de courbe de distribution) des débits moyens annuels dans les trois régimes régularisés observés (inversion, homogénéisation et type naturel) au Québec. Nous avons appliqué la méthode de proportionnalité qui consiste à comparer les caractéristiques des débits des rivières naturelles et celles des rivières régularisées en fonction de la taille des bassins versants. En ce qui concerne le volume d'écoulement-fréquence et sa variabilité interannuelle, le changement a été observé seulement en régime d'inversion. Il se traduit par une baisse des débits moyens annuels durant les années hydrologiques sèches et une variabilité interannuelle relativement forte par rapport aux rivières naturelles. Ces changements sont attribués principalement au mode de gestion des réservoirs car on lâche moins d'eau durant ces années hydrologiques sèches. Enfin, les changements des coefficients d'asymétrie et d'aplatissement ont été observés surtout en régime d'homogénéisation. Cette étude démontre que les barrages peuvent modifier toutes les caractéristiques des débits moyens annuels contrairement à l'opinion couramment admise.
Mots clés : caractéristiques des débits moyens annuels, inversion, homogénéisation, type naturel, barrages, volume d'écoulement, fréquence, variabilité interannuelle, asymétrie, aplatissement, Québec.

\section{SUMMARY}

We compared the impacts of dams on the characteristics (magnitude-frequency, inter-annual variability and distribution curve shape) of the mean annual flows in three regulated flow regimes (inversion, homogenization and natural type) in Québec. We applied the "proportionality method", which consists of comparing the flow characteristics of natural rivers to regulated rivers according to watershed size. A change in the flow-frequency volume and its inter-annual variability was observed only in the inversion flow regime. This result translates into a decrease in average annual flows during dry hydrological years and a relatively high inter-annual variability relative to natural rivers. These changes are mainly ascribed to the reservoir management mode because less water is released during dry hydrological years. Finally, the changes of

*Auteur pour correspondance :

Téléphone : (819) 376-5011

Télécopieur : (819) 376-5179

Courriel : Ali_Assani@uqtr.ca 
the coefficients of asymmetry and skewness are particularly observed in homogenization flow regime. This study shows that, contrary to the commonly accepted opinion, dams can alter all the characteristics of annual average flows.

\section{Key words: Mean annual flow characteristics, inversion, bo- mogenization, natural type, regression line, Québec.}

\section{INTRODUCTION}

Il existe une controverse relative aux impacts des barrages sur les débits moyens annuels malgré les changements des débits observés aux échelles mensuelles et journalières. Selon l'opinion couramment admise, les barrages ne modifient pas les débits moyens annuels puisque toute la quantité d'eau stockée au cours d'une année hydrologique est intégralement lâchée (ANCTIL et COULIBALY, 2004), en excluant, bien entendu, le cas de transfert inter-bassins d'eau. Cependant, certaines études ont montré que les débits moyens annuels en aval des barrages pouvaient être modifiés (e.g. BATALLA et al., 2004; CHURCH, 1995). Il convient de noter que ces études se limitent souvent à un seul bassin versant. De plus, elles n'analysent qu'une seule caractéristique des débits moyens annuels, à savoir le volume d'écoulement. Par conséquent, on ignore si les barrages peuvent modifier les autres caractéristiques malgré l'évacuation des volumes d'eau stockée durant une année hydrologique. Sur le plan écologique, ces caractéristiques jouent aussi un rôle très important dans le fonctionnement des écosystèmes aquatiques. La variabilité interannuelle est sans nul doute l'une des principales caractéristiques fondamentales des débits qui présente un intérêt majeur en écologie aquatique en raison de son influence significative sur la dynamique des populations ainsi que sur la composition et le fonctionnement des écosystèmes lotiques notamment (RICHTER et al., 1996; POFF et al., 1997; BIGGS et al., 2005). C'est ainsi qu'au Québec, par exemple, les fluctuations interannuelles des débits influencent de manière significative la biomasse et la composition spécifique de la végétation humide du fleuve Saint-Laurent (HUDON, 1997, 2004). La biomasse et le nombre d'espèces végétales augmentent durant les années sèches mais diminuent durant les années humides. De même en Nouvelle-Zélande, BIGGS et al. (1999, 2005) avaient mis en évidence l'influence de ces fluctuations interannuelles des débits sur la biomasse des périphytons et la production des invertébrés des rivières. D'autres auteurs ont démontré l'influence de la variabilité interannuelle des débits sur les populations de poissons (HAYES, 1995; McINTOCH, 2000). Les modifications qui affectent la succession des années sèches et humides peuvent modifier profondément l'équilibre des organismes en aval des barrages. Pourtant ces modifications sont plausibles du fait que l'évacuation des débits en aval des barrages ne s'effectue pas de la même façon que dans les conditions naturelles tant en ce qui concerne la fréquence que la quantité d'eau lâchée. Ainsi, dans le cas de la rivière Matawin au Québec, les périodes sèches et humides duraient plus longtemps en aval qu'en amont du barrage (ASSANI et al., 2002). Par ailleurs, une analyse comparée de la variabilité interannuelle des débits aux échelles annuelle, saisonnière et journalière en aval de quatre des barrages construits sur la rivière Saint-Maurice au Québec (Canada) a mis en évidence l'influence du mode de gestion des barrages (ASSANI, 2004). Ainsi, la variabilité interannuelle des débits était plus forte en régime d'inversion (régime caractérisé par les hautes eaux en hiver et les basses eaux au printemps) qu'en régime d'homogénéisation (les débits restent quasi constants toute l'année) et de type naturel (régime caractérisé par les hautes eaux au printemps et les basses eaux en hiver ou en été comme en rivières naturelles) aux échelles annuelle et saisonnière.

Au Québec, malgré la présence de nombreux barrages et la diversité de leur mode de gestion, aucune étude n'a jamais été entreprise pour analyser les impacts des barrages sur toutes les caractéristiques des débits moyens annuels. Notre objectif est de combler cette lacune en vérifiant les deux hypothèses suivantes :

1. La diversité de modes de gestion des barrages et de tailles des bassins versants peut entraîner des impacts différents sur les débits moyens annuels. En effet, les études sur les impacts hydrologiques déjà effectuées aux échelles mensuelle et journalière ont démontré que l'ampleur des changements hydrologiques induits par les barrages dépend $\mathrm{du}$ mode de gestion et de la taille des bassins versants au Québec (ASSANI et al., 2005, 2006; LAJOIE et al., 2007). À l'échelle journalière, les barrages provoquent une baisse significative des débits minimums et maximums annuels. Mais cette baisse est plus importante pour les petits bassins versants en ce qui concerne les débits minimums annuels, et pour les grands bassins versants, quant aux débits maximums annuels. Par ailleurs, cette diminution était plus importante en régime d'inversion que dans les deux autres régimes hydrologiques régularisés. Il était donc intéressant de vérifier si cette diminution des débits, observée aux échelles mensuelle et journalière, se répercutait à l'échelle annuelle.

2. Malgré l'évacuation de toute la quantité d'eau stockée durant une année hydrologique, le stockage d'eau dans les réservoirs, en modifiant les conditions d'écoulement naturel, peut entraîner des changements des autres caractéristiques des débits moyens annuels comme la variabilité interannuelle. Ces changements ont été observés aux échelles mensuelle et journalière. L'ampleur de ces changements dépendait du mode de gestion et de la taille des bassins versants.

Pour vérifier ces deux hypothèses, nous allons comparer les impacts de barrages dans les trois régimes hydrologiques 
artificialisés (mode de gestion) décrits au Québec en fonction de la taille des bassins versants.

\section{MÉTHODOLOGIE}

\subsection{Sources de données}

Les données des débits moyens annuels proviennent du CD-Rom HYDAT édité par ENVIRONNEMENT CANADA (1996). Nous en avons analysé 76 en rivières naturelles et 61 en rivières régularisées, affluents du fleuve Saint-Laurent (Figure 1). Les débits ont été mesurés pendant la période 1920 à 1994 . La durée des mesures de débits n'est pas la même pour toutes les rivières. En ce qui concerne les rivières régularisées, cette régularisation se limite seulement à la construction des barrages. D'autres formes d'aménagements comme le transfert inter-bassins ou l'irrigation susceptibles de modifier les caractéristiques des débits moyens annuels y sont absentes. Nous avons analysé les impacts des barrages sur les caractéristiques des débits moyens annuels. Ceux-ci peuvent être définis par quatre caractéristiques seulement (ASSANI, 2003), consignées au tableau 1 . Chaque caractéristique a été définie par au moins une variable hydrologique. Il convient de préciser que les deux caractéristiques, volume d'écoulement et fréquence, sont difficiles à différencier car elles sont exprimées par les mêmes unités, à savoir la quantité d'eau par unité de temps. Dans ce travail, en ce qui concerne le volume d'écoulement, pour chaque année hydrologique, qui commence en octobre et se termine en septembre de l'année civile suivante, nous avons d'abord calculé la moyenne arithmétique de 12 valeurs mensuelles, qu'on appelle DMA (débit moyen annuel). Ensuite, à chaque station, nous avons calculé la moyenne arithmétique des valeurs des DMA. Cette dernière moyenne a été appelée la moyenne des débits moyens annuels $\left(\mathrm{Q}_{\mathrm{mo}}\right)$. C'est cette variable qui a été utilisée pour définir le volume d'écoulement. En ce qui concerne la fréquence des débits, afin de pouvoir vérifier si les conditions hydrologiques (sèches ou humides) peuvent avoir un impact sur la gestion des barrages, nous avons retenu six variables hydrologiques: trois variables hydrologiques pour définir les années hydrologiques humides et trois autres pour définir les années hydrologiques sèches. En ce qui concerne les années hydrologiques humides, nous avons utilisé les variables suivantes : le débit maximum des DMA (Qmax, le débit moyen annuel le plus élevé), les valeurs des débits moyens annuels correspondant aux percentiles $95\left(\mathrm{Q}_{95}\right)$ et $90\left(\mathrm{Q}_{90}\right)$. Quant aux années sèches, les trois variables analysées sont : le débit minimum des DMA (Qmin, le débit moyen annuel le plus faible) ainsi que les valeurs des débits moyens annuels correspondant aux percentiles $5\left(\mathrm{Q}_{5}\right)$ et $10\left(\mathrm{Q}_{10}\right)$. Toutes les six variables

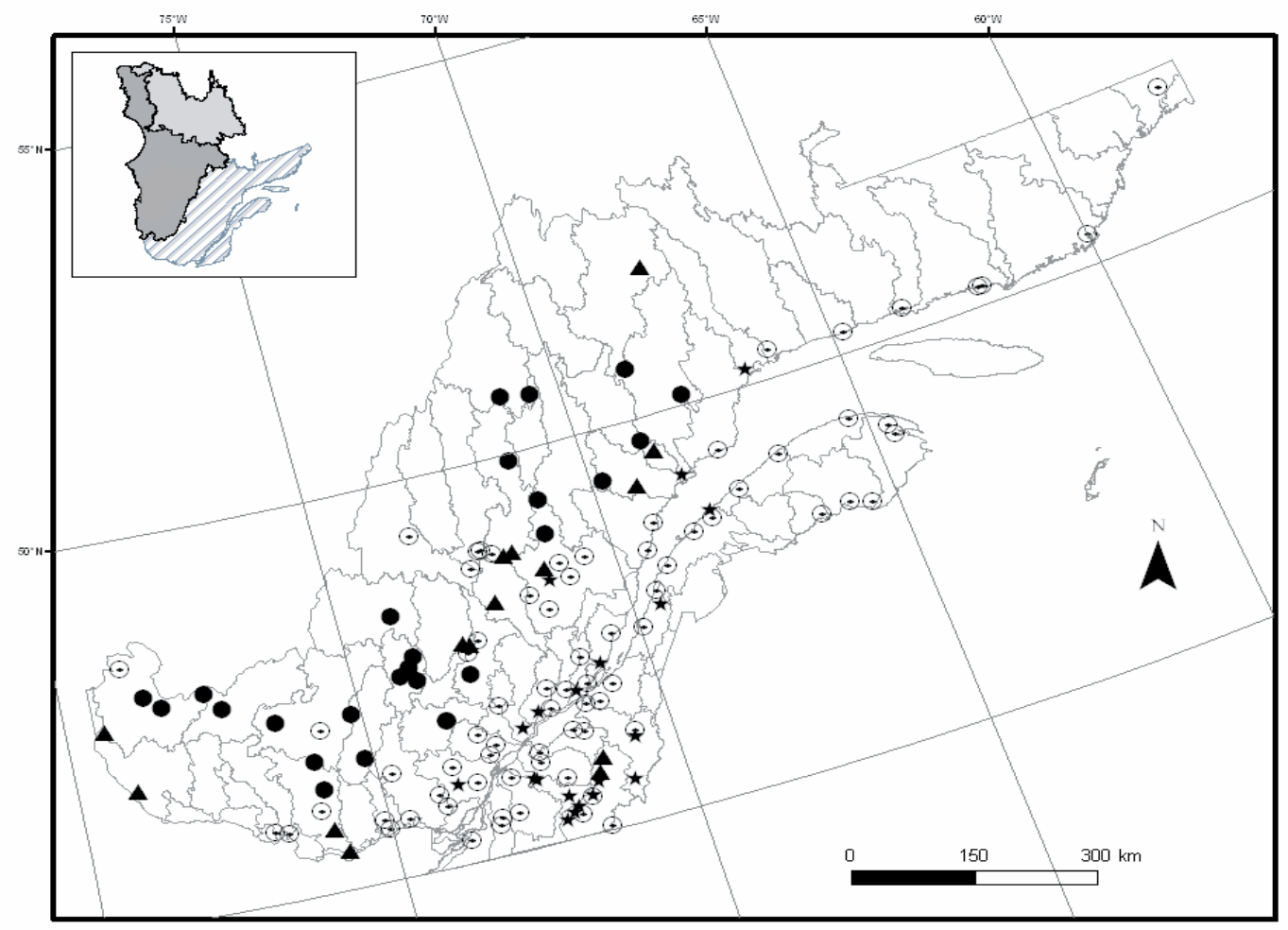

Figure 1. Localisation des stations. Cercle : rivières à régime naturel; point : rivières à régime d'inversion; triangle : rivières à régime d'homogénéisation; étoile : rivières à régime de type naturel.

Location of stations. Circle: pristine regime; point: inverted regime; triangle: homogenization regime; star: natural type regime. 
Tableau 1. Les caractéristiques et les variables statistiques des débits moyens annuels.

Table 1. Mean annual characteristics and hydrological variables.

\begin{tabular}{|c|c|c|}
\hline Caractéristiques & Variables hydrologiques & Signification et mode de calcul \\
\hline \multirow{7}{*}{ Volume d'écoulement et fréquence } & Débits maximums $\left(\mathrm{Q}_{\max }\right)$ & $\begin{array}{l}\text { Valeur des débits moyens annuels la } \\
\text { plus élevée }\end{array}$ \\
\hline & $\mathrm{Q}_{95}$ & $\begin{array}{l}\text { Valeur des débits moyens annuels } \\
\text { correspondant au percentile } 95\end{array}$ \\
\hline & $\mathrm{Q}_{90}$ & $\begin{array}{l}\text { Valeur des débits moyens annuels } \\
\text { correspondant au percentile } 90\end{array}$ \\
\hline & $\mathrm{Q}_{\mathrm{mo}}$ & $\begin{array}{l}\text { Moyenne arithmétique des débits } \\
\text { moyens annuels. }\end{array}$ \\
\hline & $\mathrm{Q}_{10}$ & $\begin{array}{l}\text { Valeur des débits moyens annuels } \\
\text { correspondant au percentile } 10\end{array}$ \\
\hline & $\mathrm{Q}_{5}$ & $\begin{array}{l}\text { Valeur des débits moyens annuels } \\
\text { correspondant au percentile } 5\end{array}$ \\
\hline & Débits minimums $\left(\mathrm{Q}_{\min }\right)$ & $\begin{array}{l}\text { Valeur des débits moyens annuels la } \\
\text { plus faible }\end{array}$ \\
\hline Variabilité inter-annuelle & $\mathrm{CV}$ & Coefficient de variation \\
\hline \multirow{2}{*}{ Forme de la courbe } & $\mathrm{C}_{\mathrm{s}}$ & Coefficient d'asymétrie \\
\hline & $\mathrm{C}_{\mathrm{k}}$ & Coefficient d'aplatissement \\
\hline
\end{tabular}

ont été déduites des séries hydrologiques analysées constituées des valeurs de DMA. Quant au coefficient de variation $(\mathrm{CV})$, qui est le rapport entre la moyenne arithmétique des DMA et son écart-type, il a aussi été calculé à partir des séries hydrologiques constituées de valeurs de DMA. Il en est de même des coefficients d'asymétrie $\left(\mathrm{C}_{\mathrm{s}}\right)$ et d'aplatissement $\left(\mathrm{C}_{\mathrm{k}}\right)$ de Pearson.

\subsection{Justification du choix de la méthode d'analyse des données}

Dans la littérature, trois méthodes sont couramment utilisées pour analyser les impacts des barrages :

- La méthode de "station témoin " qui consiste à comparer les données mesurées à la même station avant et après la construction d'un barrage (e.g. MAGILLIGAN et NISLOW, 2005).

- La méthode de "station de contrôle" fondée sur la comparaison des débits mesurés en amont et en aval d'un barrage (e.g. ASSANI et al., 2002) ou sur la comparaison des débits mesurés sur une rivière non influencée par un barrage et en aval d'un barrage (e.g. BENN et ERSKINE, 1994).

- La méthode de « reconstitution" qui consiste à comparer les débits reconstitués en condition naturelle et les débits lâchés en aval d'un barrage. Cette reconstitution des débits peut être effectuée à partir de la production journalière de l'énergie électrique (e.g. ASSANI et al., 1999) ou au moyen d'un modèle hydrologique (e.g. MAHESHWARI et al., 1995; PETERS et PROWSE, 2001).

Dans le cadre de cette étude, nous ne pouvions appliquer aucune de ces trois méthodes pour les raisons suivantes :

- Ces trois méthodes ne permettent pas de mettre en évidence l'influence du mode de gestion des barrages et de la taille des bassins versants sur l'ampleur des modifications hydrologiques induites par les barrages du fait qu'elles consistent à analyser les stations séparément. Or, pour mettre en évidence l'influence de ces facteurs, il faut absolument comparer simultanément les bassins versants de différentes tailles. Ainsi, l'utilisation de l'une de ces trois méthodes ne nous permettrait pas de vérifier les deux hypothèses sur lesquelles se fonde notre étude.

- Nous ne disposions pas de données de débits ni avant la construction des barrages ni en amont des barrages. Par conséquent, on ne pouvait pas appliquer les deux premières méthodes.

- Il est impossible de simuler les débits naturels au moyen d'un modèle hydrologique unique pour toutes les stations 
analysées. De plus, l'élaboration d'un modèle hydrologique est un processus long et fastidieux et ne peut se justifier dans le cadre de ce travail. C'est une méthode généralement utilisée lorsqu'on travaille sur une seule station ou sur plusieurs stations d'une même rivière.

Nous avons ainsi utilisé une nouvelle méthode d'analyse d'impacts de barrages qui consiste à comparer les débits en rivières naturelles et les débits en rivières influencées par les barrages en fonction de la taille des bassins versants (méthode de proportionnalité). Cette approche se justifie du fait qu'en rivières naturelles, il existe une forte proportionnalité entre les débits (annuels, mensuels et journaliers) et les superficies de bassins versants au Québec (ASSANI et al., 2005, 2006; BELZILE et al., 1997; LAJOIE et al., 2007) malgré l'influence des autres facteurs physiographiques. À titre d'exemple, la figure 2 présente la relation entre le nombre d'années de mesure des débits et deux variables hydrologiques qui sont la moyenne (MDMA) et les coefficients de variation (CV) des débits moyens annuels de rivières non régularisées. Dans les deux cas, la valeur du coefficient de détermination calculée entre les deux variables est inférieure à $1 \%$. En d'autres termes, le nombre d'années de mesure n'a aucune influence sur la variabilité spatiale des débits moyens annuels. Il en est de même des coefficients de variation. L'absence de l'influence du nombre d'années de mesure sur les caractéristiques des débits moyens annuels nous autorise à tenir compte de toutes les stations indépendamment de la durée et de la période de mesure des débits aussi bien en rivières naturelles qu'en rivières régularisées. Toutefois, pour des raisons statistiques, nous avons retenu des stations dont les mesures des débits s'étendent sur au moins 10 ans, seuil minimum considéré comme suffisant pour les analyses statistiques en hydrologie.

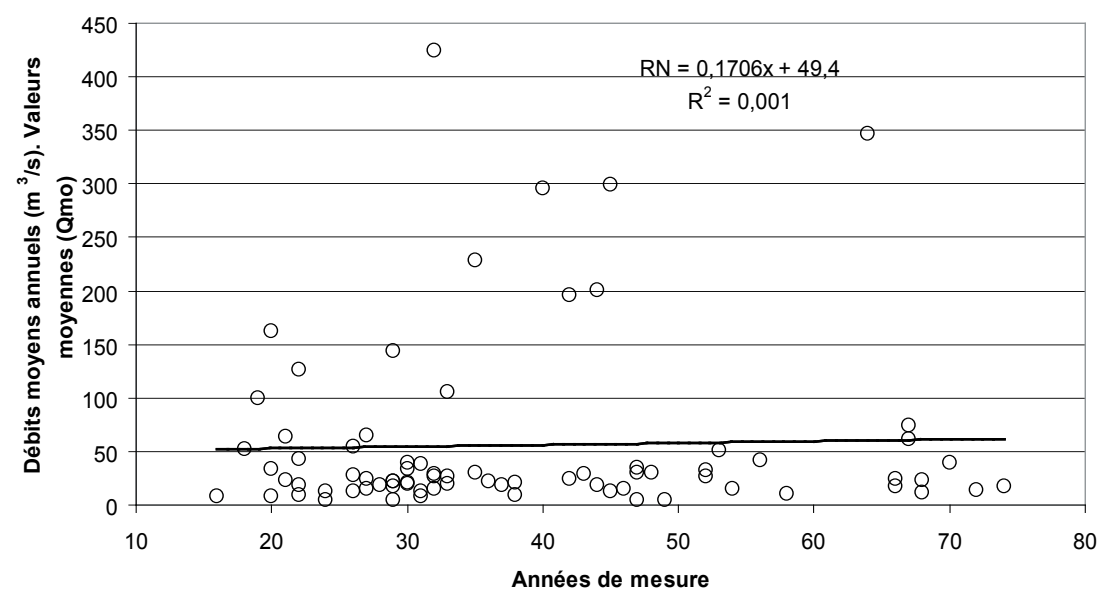

(a)

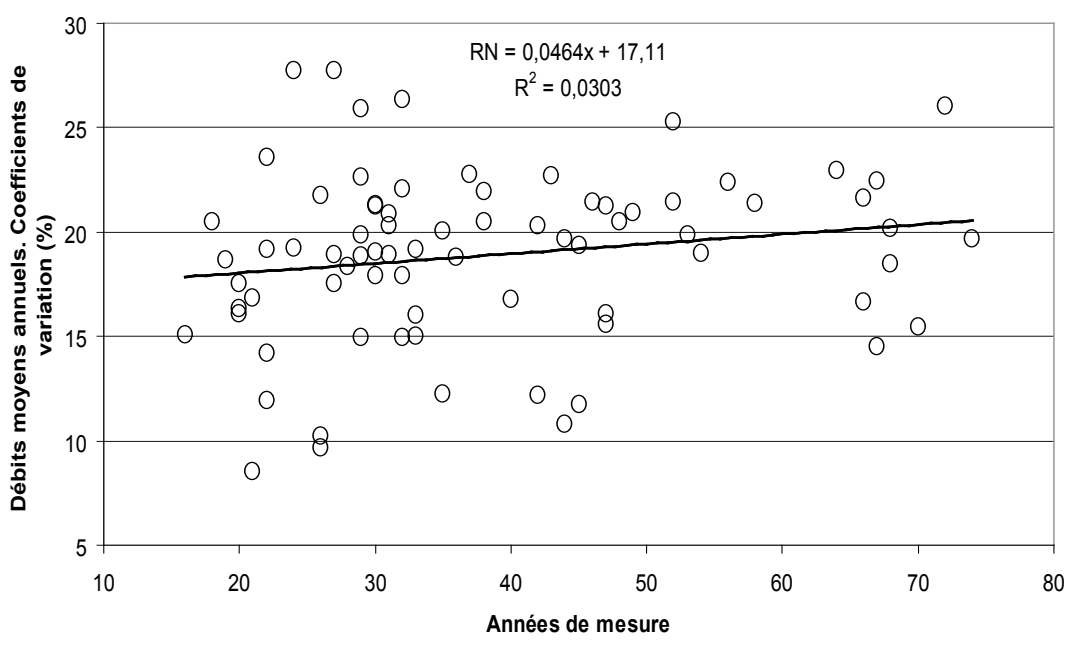

(b)

Figure 2. Relation entre le nombre d'années et (a) les moyennes arithmétiques des débits moyens annuels et (b) les coefficients de variation des débits moyens annuels en rivières naturelles.

Relationship between the number of years and (a) mean and (b) coefficients of variation of MAF in pristine rivers. 


\subsection{Description de la méthode de proportionnalité}

La méthode de proportionnalité utilisée dans cette étude pour comparer les débits de rivières régularisées et non régularisées comprend deux étapes importantes :

1. La première étape consiste à calculer deux régressions, après transformation logarithmique, entre les débits moyens annuels et les superficies de bassins versants pour les rivières non régularisées, d'une part, et pour les rivières régularisées, d'autre part. La significativité de chaque droite de régression est testée par la méthode de l'analyse de variance. Si les deux régressions sont statistiquement significatives, on compare leurs paramètres (pente et ordonnée à l'origine) au moyen de l'analyse de variance ou du test t de Student. L'objectif de cette étape est de pouvoir déterminer si la relation entre les débits et la superficie des bassins versants est significativement différente en rivières naturelles et en rivières régularisées. C'est cette étape qui permet de déterminer l'influence du mode de gestion des barrages et de la taille des bassins versants sur les caractéristiques des débits.

2. Lorsque les deux régressions sont significativement différentes, la seconde étape consiste à quantifier l'ampleur du changement (hausse ou baisse) qui affecte les débits en rivières régularisées. Cette quantification consiste à analyser séparément les stations. Pour quantifier ce changement, nous avons procédé selon les étapes suivantes (ASSANI et al., 2005, 2006) :

- Nous avons comparé les débits moyens annuels observés $\left(Q_{0}\right)$ à chaque station influencée par les barrages aux débits moyens annuels estimés $\left(\mathrm{Q}_{\mathrm{e}}\right)$, à partir des superficies des bassins versants, par la régression établie entre les débits et la taille des bassins versants en rivières naturelles. Le débit moyen annuel estimé $\left(\mathrm{Q}_{\mathrm{e}}\right)$ correspond théoriquement au débit moyen annuel qui devrait être observé à une station sans la présence d'un barrage. Cette estimation se justifie par le fait qu'il existe une très forte proportionnalité entre les débits moyens annuels et les superficies de bassins versants en condition naturelle comme nous le verrons plus loin.

- Ensuite, nous avons calculé les intervalles de confiance des $Q_{e}$ au seuil de $95 \%$.

- Enfin, à chaque station, nous avons comparé les valeurs des $\mathrm{Q}_{\mathrm{o}}$ et $\mathrm{Q}_{\mathrm{e}}$ en tenant compte, bien entendu, de l'intervalle de confiance de ce dernier. Lorsque le débit mesuré en aval d'un barrage $\left(\mathrm{Q}_{\mathrm{o}}\right)$ se situait à l'intérieur de cet intervalle de confiance, nous avons donc considéré que les deux débits $\left(Q_{e}\right.$ et $\left.Q_{o}\right)$ n'étaient pas significativement différents même si les régressions ajustées sur l'ensemble des stations le sont. Ainsi, il n'y a aucun changement significatif des débits induit par le barrage à cette station. On conçoit aisément que l'ampleur des impacts hydrologiques ne peut être la même pour tous les barrages même si ceux-ci sont soumis au même mode de gestion.

À première vue, la méthode de proportionnalité peut paraître relativement moins précise que la méthode de "station témoin ", par exemple, dans la quantification des changements de débits induits par les barrages. En fait, ce n'est point le cas. En effet, elle permet aussi de quantifier avec précision le changement qui affecte le débit en aval d'un barrage au moyen d'un test statistique rigoureux par le calcul d'intervalles de confiance comme nous venons de le démontrer. De plus, son avantage incontestable par rapport aux trois autres méthodes est de pouvoir analyser simultanément les bassins versants de différentes tailles (étape 1). On peut ainsi déterminer à la fois l'influence de la taille des bassins versants et le mode de gestion des barrages sur l'ampleur des changements induits par ceux-ci sur les débits. Par ailleurs, elle présente plusieurs points communs avec les trois autres méthodes. À l'instar de la méthode de "station témoin ", elle permet de quantifier de manière précise les changements des débits induits par un barrage comme nous venons de le souligner. Elle utilise les données de débits qui ne sont pas mesurées sur la même rivière sur laquelle est construit un barrage comme la méthode de station contrôle. Enfin, elle utilise les données des débits estimés en condition naturelle pour quantifier les changements hydrologiques comme la méthode de reconstitution.

En cas d'absence de proportionnalité ou de faible proportionnalité (lorsque les deux ou une de deux régressions ajustées sur les données ne sont pas statistiquement significatives) entre une variable hydrologique et la superficie des bassins versants, nous avons procédé par une simple comparaison graphique (méthode graphique). Cette approche est aussi utilisée dans le cadre des trois autres méthodes susmentionnées. Elle permet d'évaluer de manière qualitative (hausse ou baisse de la variable hydrologique analysée) les changements induits par un barrage lorsqu'une évaluation quantitative ne peut être appliquée.

\subsection{Description des régimes hydrologiques régularisés}

En ce qui concerne le mode de gestion des barrages au Québec, nous avons mis en évidence trois régimes hydrologiques artificialisés à partir des débits mensuels et saisonniers (ASSANI et al., 2005, 2006). Chaque régime hydrologique artificialisé correspond à un mode de gestion spécifique.

- Le régime d'inversion est le régime le plus affecté par les barrages car toutes les caractéristiques des débits sont modifiées. Le régime est caractérisépar unehaussesignificative des débits saisonniers hivernaux, estivaux et automnaux et des débits mensuels minimums, d'une part, et une baisse 
significative des débits printaniers et des débits mensuels maximums, d'autre part. On y observe un changement des mois d'occurrence des débits mensuels maximums et minimums, c'est-à-dire que les débits mensuels maximums surviennent en hiver et les débits mensuels minimums au printemps, donc l'inverse de ce qui se produit en condition naturelle. Il y a donc une inversion du cycle naturel des débits. Ce régime s'observe exclusivement sur la rive nord $\mathrm{du}$ bassin du fleuve Saint-Laurent en aval des réservoirs afin d'alimenter les centrales hydroélectriques construites en aval en période hivernale.

- Le régime d'homogénéisation est caractérisé par une uniformisation des débits mensuels et saisonniers durant toute l'année. Les débits maximums surviennent souvent au printemps mais parfois en hiver. En revanche, contrairement au régime précédent, les débits minimums mensuels et saisonniers ne surviennent jamais au printemps. La variation inter-mensuelle des débits est très faible comparativement au régime précédent. Les débits restent ainsi presque constants toute l'année. Ce régime est aussi presque exclusivement observé sur la rive nord du fleuve Saint-Laurent. Il est associé généralement aux bassins versants de grande taille $\left(>10000 \mathrm{~km}^{2}\right)$.

- Le régime de type « naturel » est le régime le moins modifié par les barrages. Toutefois, toutes les caractéristiques des débits sont modifiées mais avec moins d'ampleur par rapport aux deux autres régimes. Tout d'abord, on observe une légère hausse des débits hivernaux et des débits mensuels minimums mais une baisse modérée des débits printaniers et des débits mensuels maximums. En ce qui concerne la période d'occurrence des débits, le changement affecte seulement la période des débits mensuels minimums qui arrive fréquemment en été. Ce régime est associé aux barrages construits au fil de l'eau et qui sont presque tous dotés de centrales hydroélectriques.

\section{RÉSULTATS}

\subsection{Impacts des barrages sur le volume d'écoulement et la distribution des débits moyens annuels}

La figure 3 compare les valeurs moyennes des débits moyens annuels entre les rivières naturelles et les rivières régularisées de trois régimes hydrologiques artificialisés. Les régressions ajustées sur les rivières naturelles et les rivières régularisées sont statistiquement significatives. La comparaison des paramètres (pente et ordonné à l'origine) de ces régressions au moyen de l'analyse de variance n'a révélé aucune différence significative (Tableau 2). La figure 3 montre clairement que les régressions et les débits de rivières non régularisées et régularisées se superposent. À la lumière de ces résultats, nous pouvons donc conclure que les barrages ne modifient pas significativement les moyennes des débits moyens annuels au Québec. Par conséquent, le rapport entre les moyennes des débits moyens annuels et la superficie des bassins versants est conservé en aval des barrages.

Pour déterminer l'influence des conditions hydrologiques, nous avons comparé aussi les débits maximums (années hydrologiques humides), d'une part (Figure 4), et les débits minimums (années hydrologiques sèches), d'autre part (Figure 5). En ce qui concerne les débits maximums (Qmax), aucune différence significative n'a été observée entre les rivières non régularisées et les rivières régularisées comme le confirme le test de l'analyse de variance (Tableau 2). Par conséquent, les barrages ne modifient pas les débits moyens annuels pendant les années hydrologiques humides. En revanche, pour les débits minimums (Qmin), une différence significative a été observée en régime d'inversion. Il se produit donc un changement significatif des débits moyens annuels en aval de ce type de barrage. Ce changement se traduit par une baisse significative des débits (Figure 5a). Pour confirmer ces résultats, nous avons analysé d'autres percentiles qui définissent les années humides $\left(\mathrm{Q}_{95}\right.$ et $\left.\mathrm{Q}_{90}\right)$ et sèches $\left(\mathrm{Q}_{10}\right.$ et $\left.\mathrm{Q}_{5}\right)$. Les résultats de ces comparaisons, consignés dans le tableau 2, confirment la différence significative entre les rivières naturelles et les rivières régularisées en régime d'inversion. En effet, l'analyse de variance a révélé des différences statistiquement significatives entre les régressions ajustées sur les rivières non régularisées et les rivières régularisées en régime d'inversion pendant les années hydrologiques sèches $\left(\mathrm{Q}_{5}\right.$ et $\left.\mathrm{Q}_{10}\right)$. En aval des réservoirs caractérisés par un régime d'inversion, on observe une baisse significative des débits moyens annuels durant les années sèches. Pour les deux autres régimes hydrologiques artificialisés, l'influence des barrages se traduit par une baisse des valeurs de coefficients de détermination $\left(\mathrm{R}^{2}\right)$. En aval des barrages, la valeur de $\mathrm{R}^{2}$ devient inférieure à $90 \%$. Il s'ensuit que les barrages affectent le rapport entre les débits moyens annuels et la superficie des bassins versants.

Pour quantifier la baisse observée des débits en régime d'inversion, nous avons comparé les valeurs de débits moyens annuels minimums observés $\left(Q_{0}\right)$ et de débits moyens annuels minimums estimés $\left(Q_{e}\right)$ selon la démarche décrite dans la partie méthodologique. Pour environ $35 \%$ des stations, les valeurs de $Q_{e}$ et de $Q_{0}$ sont significativement différentes car $\mathrm{Q}_{\mathrm{o}}$ se situe à l'extérieur de l'intervalle de confiance de $\mathrm{Q}_{\mathrm{e}}$. L'écart le plus élevé entre $Q_{e}$ et $Q_{0}$ a été observé en aval du réservoir de la rivière Manicouagan (Manic V). La valeur de $\mathrm{Q}_{\mathrm{o}}$ ne représente que $5 \%$ de celle de $\mathrm{Q}_{\mathrm{e}}$, soit une diminution de l'ordre de $95 \%$. Il faut cependant noter que cette valeur de $Q_{0}$ a été enregistrée une année après la construction du réservoir. Cette importante réduction serait attribuable au remplissage du réservoir qui est parmi l'un des plus importants 


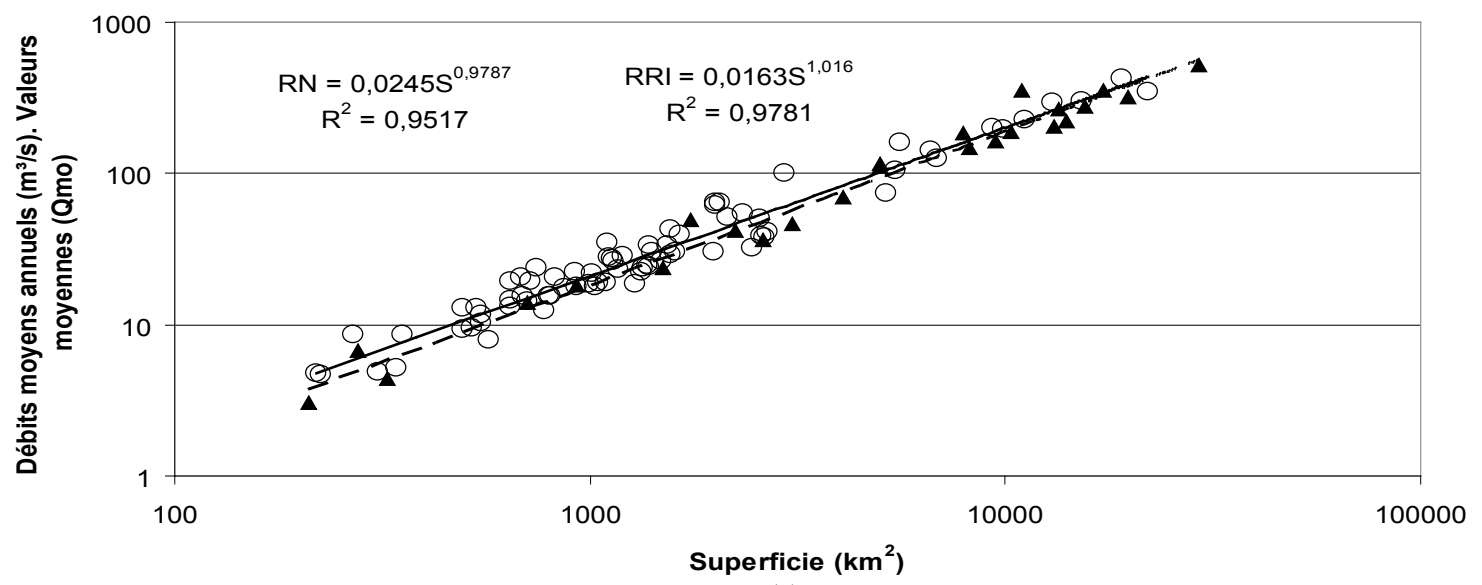

(a)

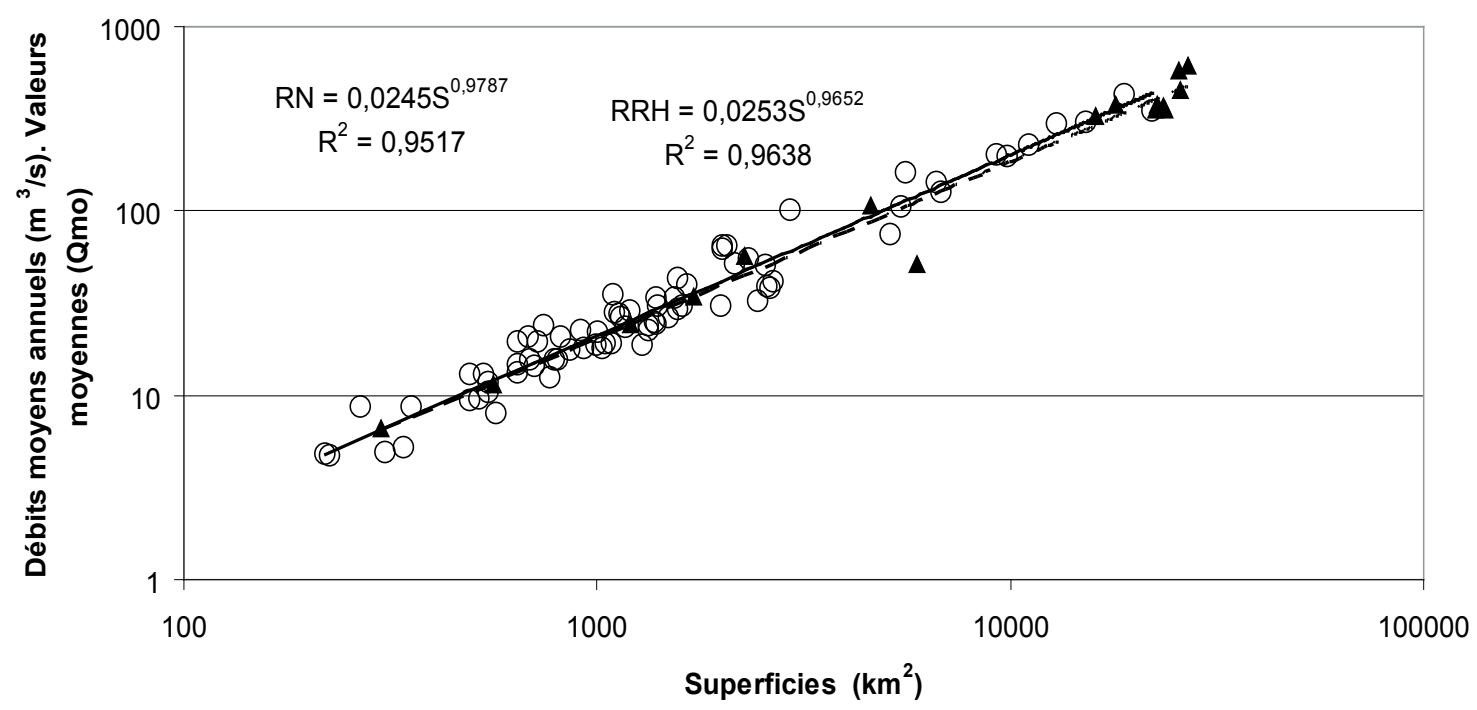

(b)

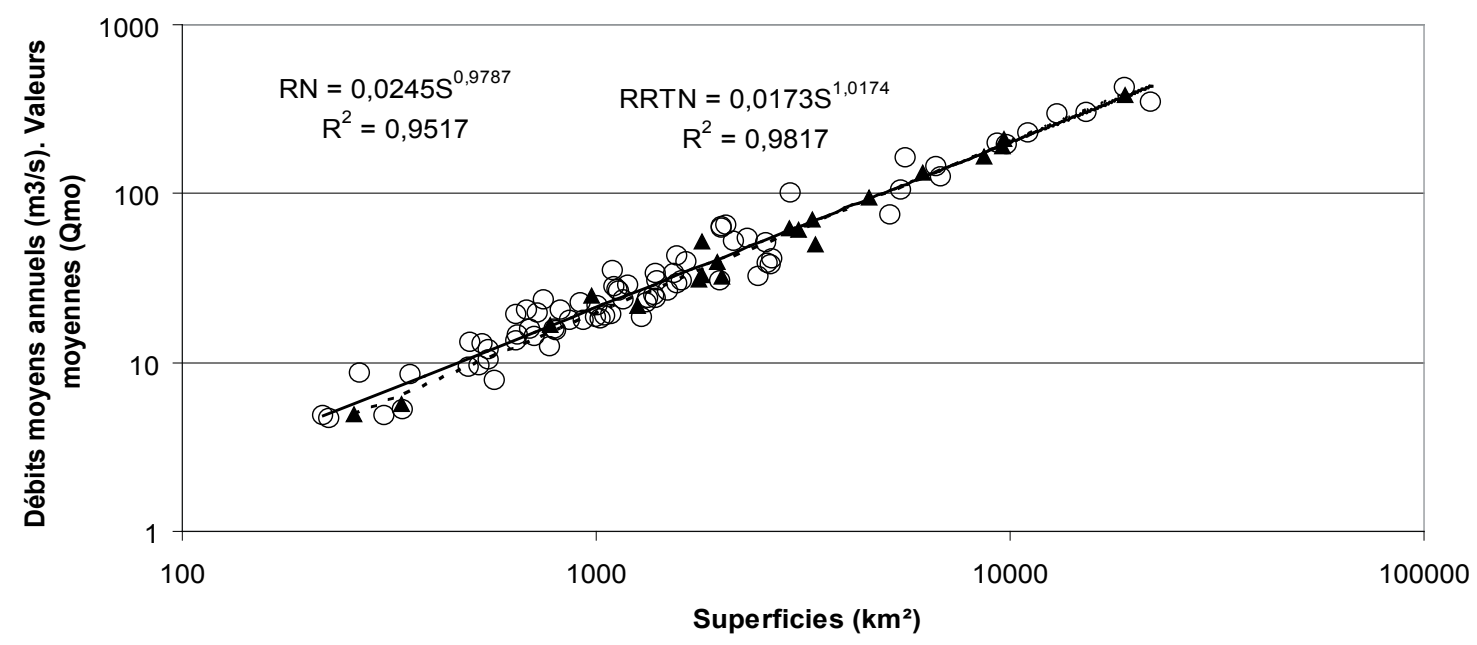

(c)

Figure 3. Comparaison de la relation entre superficies des bassins versants et moyennes arithmétiques des débits moyens annuels en rivières naturelles (cercles et ligne continue, $\mathrm{RN}$ ) et régularisées (triangles et lignes discontinues, RR); (a) régime d'inversion; (b) régime d'homogénéisation; (c) régime de type naturel. Comparison of the relationship between drainage area and arithmetic mean of MAF magnitudefrequencies in unregulated (open circles and unbroken line, $R N$ ) and regulated rivers (triangles and broken lines, RR); (a) inversed regime; (b) homogenization regime; (c) natural type regime. 


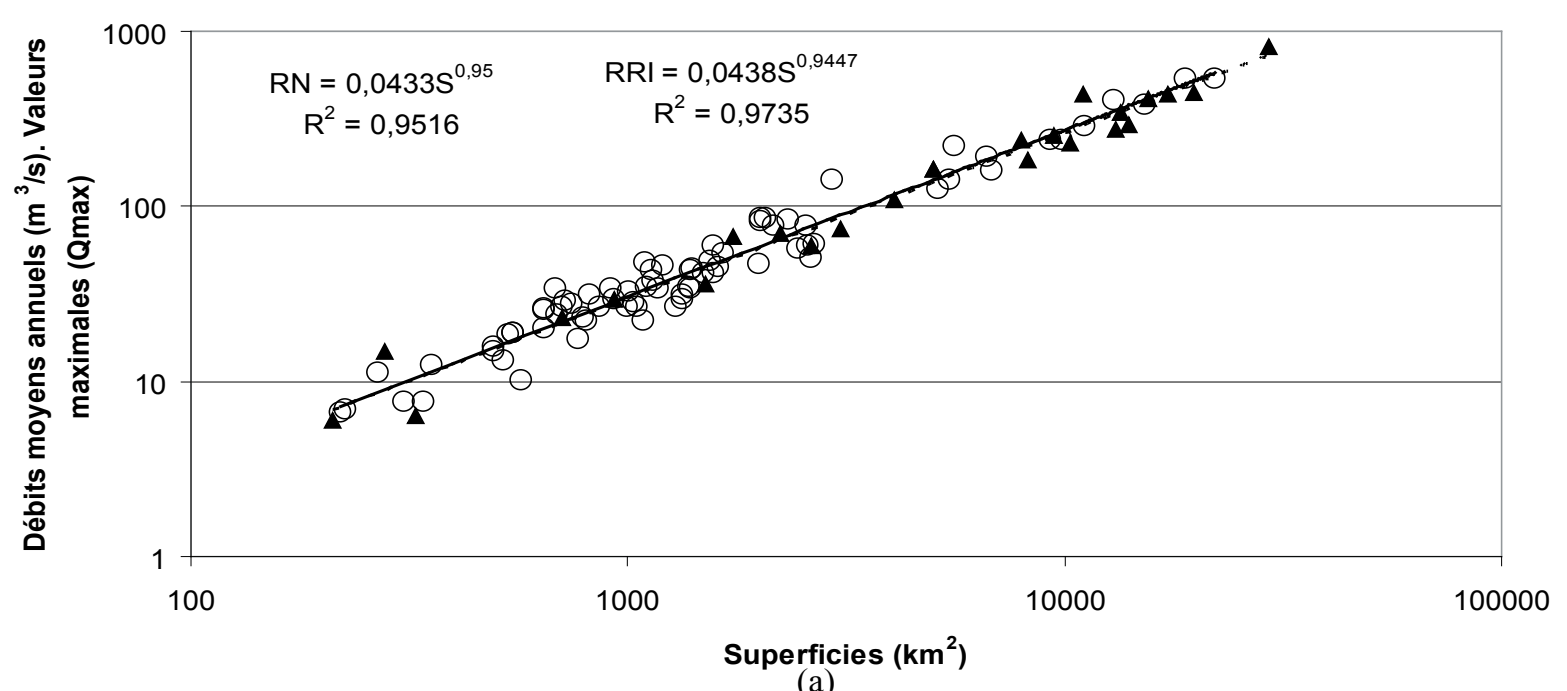

(a)

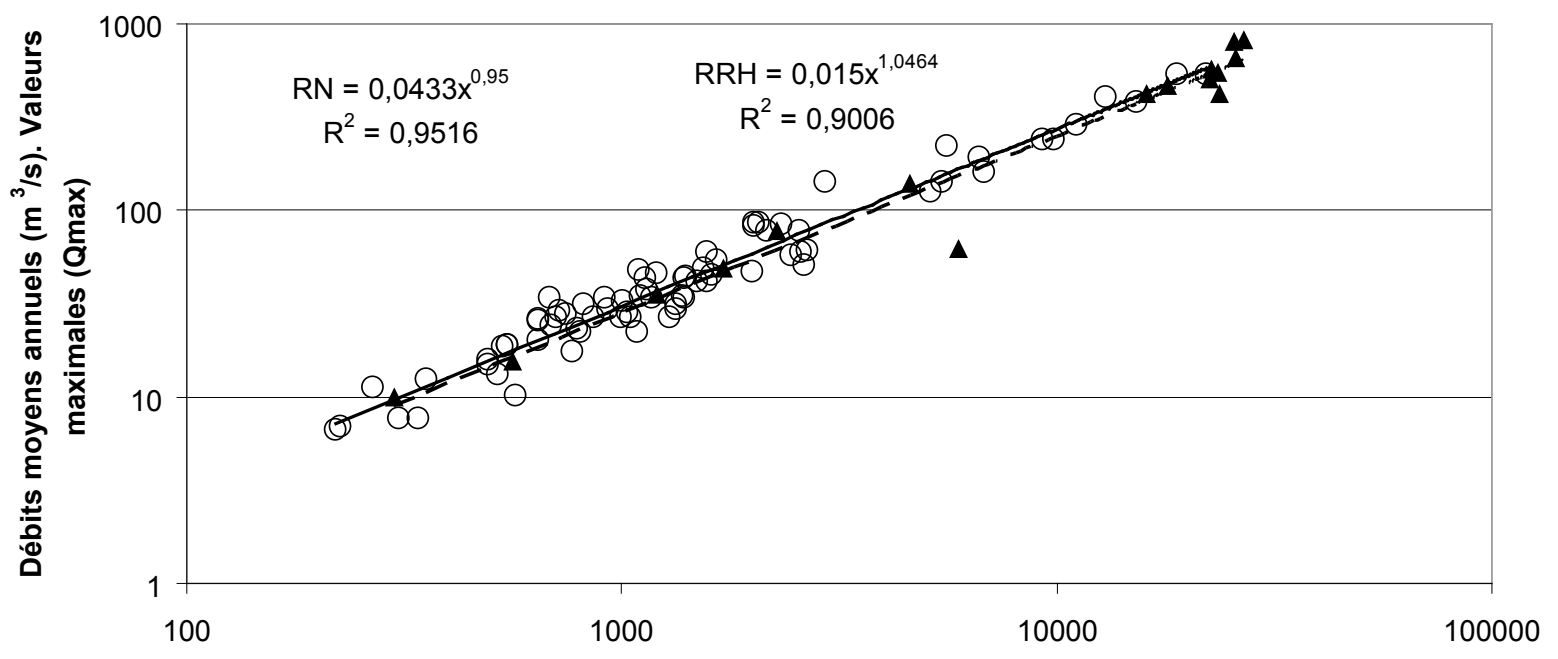

Superficies $\left(\mathrm{km}^{2}\right)$

(b)

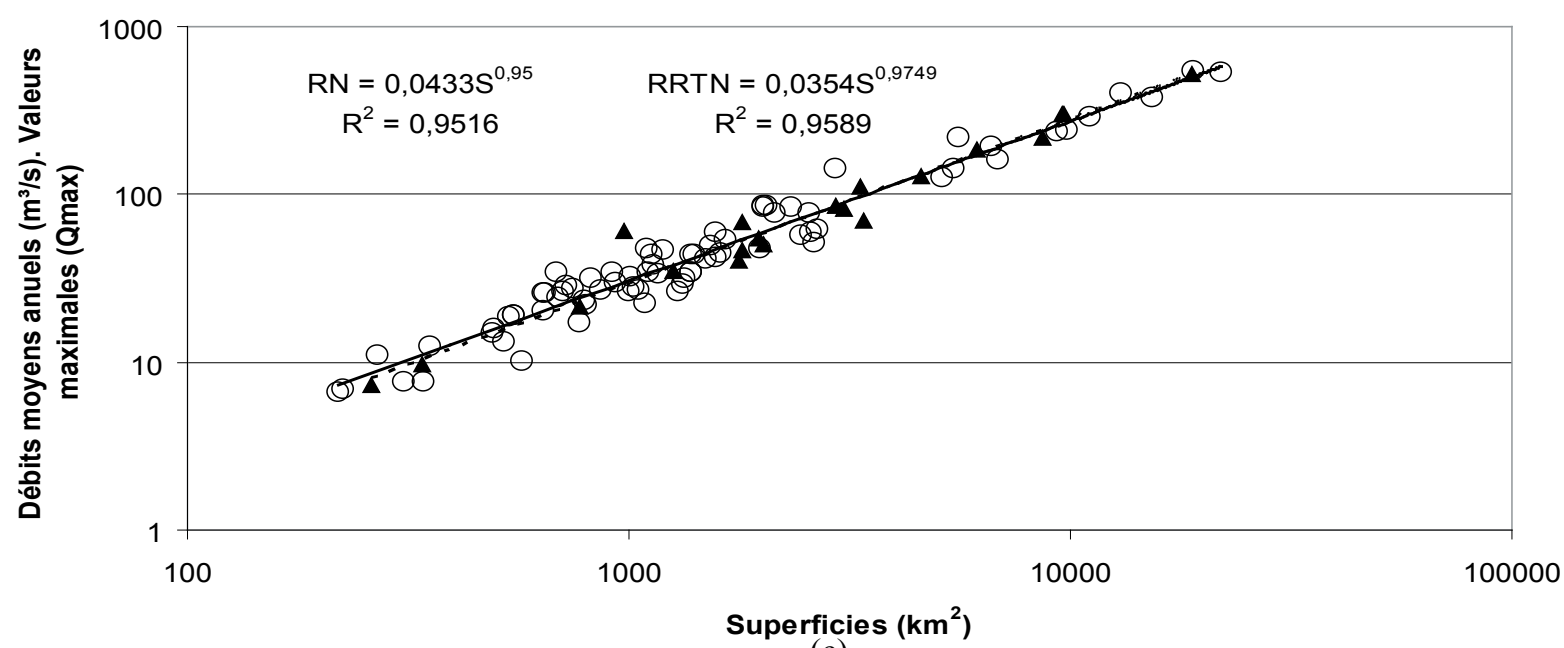

(c)

Figure 4. Comparaison de la relation entre superficies des bassins versants et valeurs maximales (Qmax) des débits moyens annuels en rivières naturelles (cercles et ligne continue, $\mathrm{RN}$ ) et rivières régularisées (triangles et ligne discontinue, $\mathrm{RR}$ ); (a) régime d'inversion; (b) régime d'homogénéisation; (c) régime de type naturel.

Comparison of the relationship between drainage area and maximum MAF magnitudes in unregulated (open circles and unbroken line, $R N$ ) and regulated rivers (triangles and broken lines, RR); (a) inversed regime; (b) homogenization regime; (c) natural type regime. 

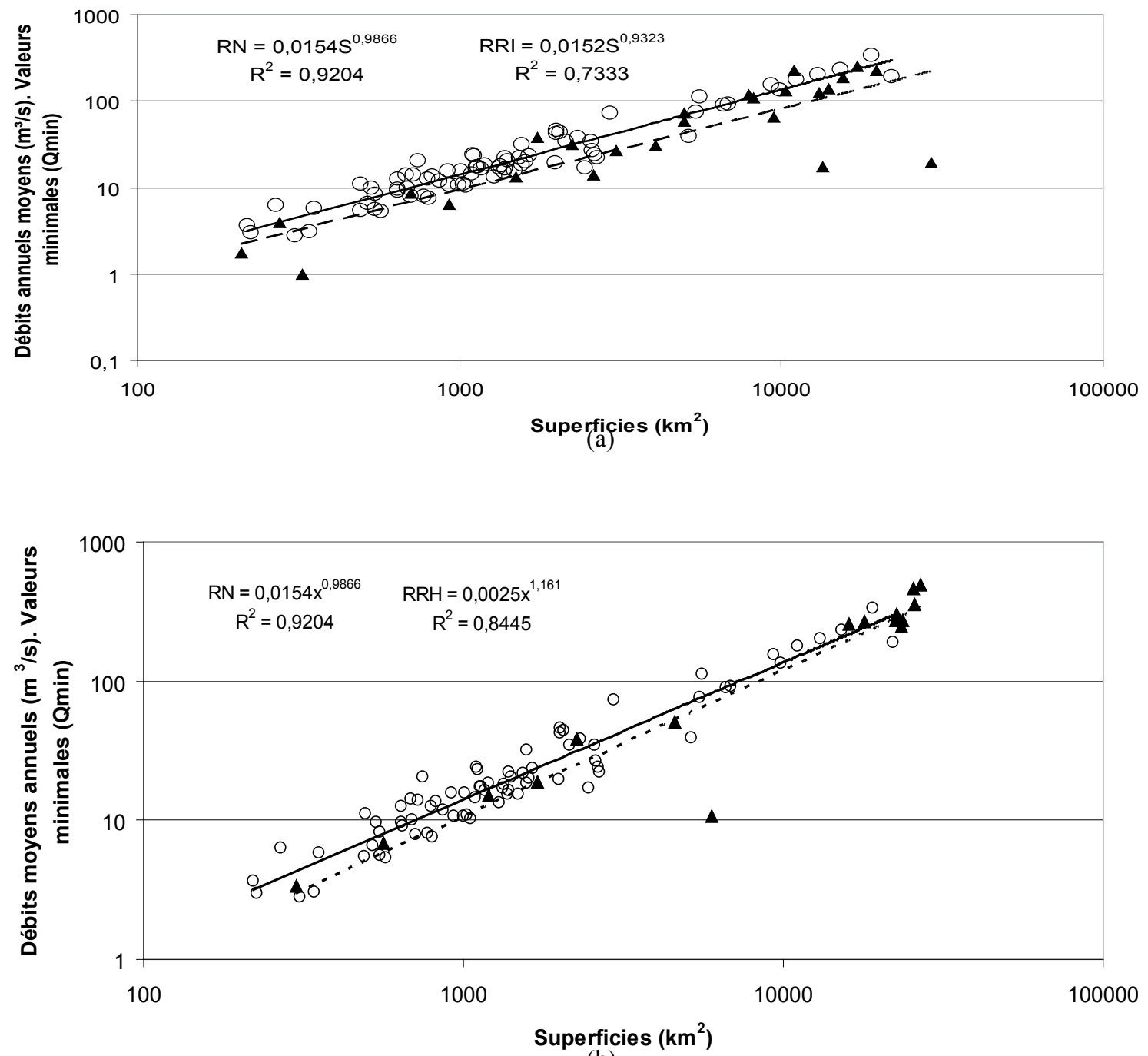

(b)

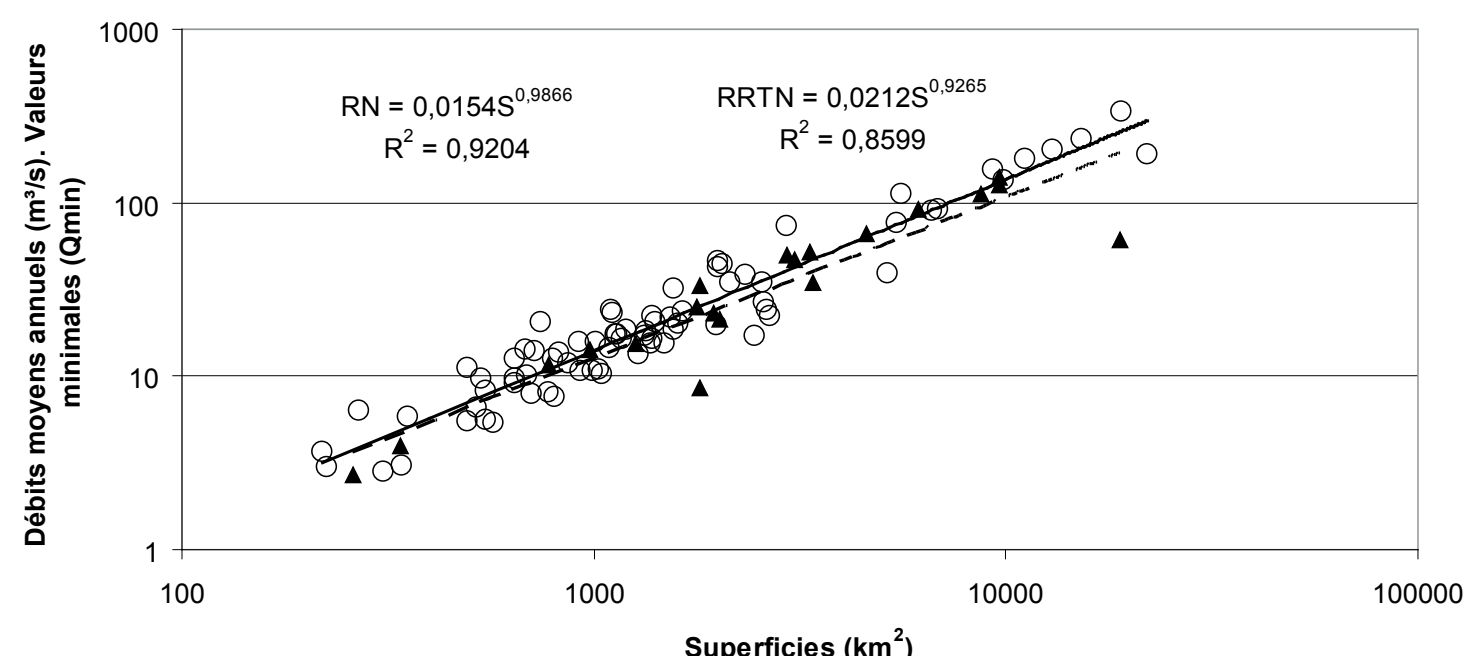

(c)

Figure 5. Comparaison de la relation entre superficies des bassins versants et valeurs minimales (Qmin) des débits moyens annuels en rivières naturelles (cercles et ligne continue, $\mathrm{RN}$ ) et régularisées (triangles, et lignes discontinues, $\mathrm{RR}$ ); (a) régime d'inversion; (b) régime d'homogénéisation; (c) régime de type naturel. Comparison of the relationship between drainage area and minimum MAF magnitudes in unregulated (open circles and unbroken line, $R N$ ) and regulated rivers (triangles and broken lines, $R R$ ); (a) inversed regime; (b) bomogenization regime; (c) natural type regime. 
Tableau 2. Comparaison des paramètres de régressions. Résultats de l'analyse de variance (test de parallélisme).

Table 2. Comparison of regression parameters. Results of analysis of variance.

\begin{tabular}{|c|c|c|c|c|}
\hline $\begin{array}{c}\text { Variables } \\
\text { hydrologiques }\end{array}$ & & $\begin{array}{c}\text { Inversion } \\
(2,97)^{*}\end{array}$ & $\begin{array}{c}\text { Homogénéisation } \\
(2,88)^{*}\end{array}$ & $\begin{array}{c}\text { Type Naturel } \\
(2,92)^{*} \\
\end{array}$ \\
\hline \multirow{2}{*}{$\mathrm{Q}_{\max }$} & $\mathrm{F}$ & 0,15 & 0,69 & 0,12 \\
\hline & $\mathrm{p}>\mathrm{F}$ & 0,8598 & 0,5044 & 0,8842 \\
\hline \multirow{2}{*}{$\mathrm{Q}_{95}$} & $\mathrm{~F}$ & 0,86 & 1,45 & 0,25 \\
\hline & $\mathrm{p}>\mathrm{F}$ & 0,4254 & 0,2398 & 0,7805 \\
\hline \multirow{2}{*}{$\mathrm{Q}_{90}$} & $\mathrm{~F}$ & 1,31 & 1,22 & 0,35 \\
\hline & $\mathrm{p}>\mathrm{F}$ & 0,2748 & 0,3013 & 0,7037 \\
\hline \multirow{2}{*}{$\mathrm{Q}_{\mathrm{mo}}$} & $\mathrm{F}$ & 2,24 & 0,55 & 0,63 \\
\hline & $\mathrm{p}>\mathrm{F}$ & 0,1125 & 0,5818 & 0,5363 \\
\hline \multirow{2}{*}{$\mathrm{Q}_{10}$} & $\mathrm{~F}$ & 4,24 & 0,53 & 0,40 \\
\hline & $\mathrm{p}>\mathrm{F}$ & 0.0172 & 0,5907 & 0,6707 \\
\hline \multirow{2}{*}{$\mathrm{Q}_{5}$} & $\mathrm{~F}$ & 4,92 & 0,63 & 0,76 \\
\hline & $\mathrm{p}>\mathrm{F}$ & 0,0092 & 0,5347 & 0,4685 \\
\hline \multirow{2}{*}{$\mathrm{Q}_{\min }$} & $\mathrm{F}$ & 7,25 & 1,79 & 1,77 \\
\hline & $\mathrm{p}>\mathrm{F}$ & 0,0012 & 0,1729 & 0,1769 \\
\hline
\end{tabular}

$*(\mathrm{a}, \mathrm{b})=$ nombre de degrés de liberté pour le test $\mathrm{F} . \mathrm{a}=$ numérateur et $\mathrm{b}=$ dénominateur. $\mathrm{p}=$ seuil de signification du test $\mathrm{F}$. Les valeurs de F statistiquement significatives au seuil de $5 \%$ apparaissent en gras.

au monde. Quoi qu'il en soit, si on considère les valeurs des débits correspondant aux percentiles $5\left(\mathrm{Q}_{5}\right)$ et $10\left(\mathrm{Q}_{10}\right)$, on observe une diminution des débits respectivement de $70 \%$ et $53 \%$. Durant les années sèches, les débits moyens annuels lâchés en aval du réservoir Manic $\mathrm{V}$ représentent moins de la moitié des débits qui devraient être mesurés en condition naturelle. Il en est de même des autres rivières comme Mondonac ou des Outaouais. En conclusion, cette étude démontre de manière évidente que les barrages peuvent provoquer une baisse significative des débits moyens annuels lors des années hydrologiques sèches au Québec. Mais cette baisse n'affecte que les barrages caractérisés par un régime d'inversion dont les débits peuvent diminuer de plus de la moitié pour certaines rivières.

\subsection{Impacts des barrages sur la variabilité interannuelle des débits}

Les valeurs de coefficients de variation sont présentées à la figure 6. Notons d'emblée qu'il n'existe aucun lien significatif entre ces valeurs et la taille des bassins versants aussi bien en rivières naturelles qu'en rivières régularisées. Néanmoins, en rivières naturelles, on peut observer que pour les rivières de taille inférieure à $5000 \mathrm{~km}^{2}$, les coefficients de variation sont globalement plus élevés que ceux des rivières de taille supérieure à ce seuil. De plus, la valeur maximale du coefficient de variation pour les premières rivières est d'environ $30 \%$ alors qu'elle ne dépasse pas $25 \%$ pour les dernières rivières. Quant aux rivières régularisées, on observe de manière évidente qu'en régime d'inversion, les deux valeurs seuils sont dépassées par plusieurs rivières. Par conséquent, ce régime est caractérisé par une variabilité interannuelle des débits relativement plus forte qu'en régime naturel. Pour les deux autres régimes régularisés, nous n'avons observé aucune différence notable entre les rivières naturelles et les rivières régularisées.

À l'instar du volume d'écoulement, les changements les plus apparents de la variabilité interannuelle des débits moyens annuels sont observés seulement en régime d'inversion. Ces changements se traduisent par une variabilité interannuelle relativement plus forte qu'en rivières naturelles.

\subsection{Impacts des barrages sur la forme de la courbe de distribution des débits moyens annuels}

Rappelons que les formes des courbes de distribution ont été définies par les coefficients d'asymétrie $\left(\mathrm{C}_{\mathrm{s}}\right)$ et d'aplatissement $\left(\mathrm{C}_{\mathrm{k}}\right)$ de Pearson. En ce qui concerne les valeurs de coefficients d'asymétrie, elles sont présentées à la figure 7 . Il ressort de cette figure que les changements les plus importants affectent surtout le régime d'homogénéisation (Figure $7 \mathrm{~b}$ ). Ce changement dépend de la taille des bassins versants. Pour les bassins versants de taille supérieure à $10000 \mathrm{~km}^{2}$, on observe globalement une hausse des valeurs des coefficients d'asymétrie en aval des barrages où la valeur de 0,5 est dépassée contrairement aux rivières non régularisées. Pour les bassins versants de taille comprise entre 5000 et $10000 \mathrm{~km}^{2}$, on observe plutôt une baisse des valeurs de coefficients d'asymétrie, qui deviennent inférieures à -1. Il faut cependant noter qu'on dispose seulement de deux stations en aval du barrage. Pour les stations de taille inférieure à $5000 \mathrm{~km}^{2}$, aucun changement n'a été observé. En régime d'inversion (Figure $7 \mathrm{a}$ ), on doit surtout noter une tendance à la baisse des valeurs de coefficients d'asymétrie pour les grands bassins versants $\left(10000 \mathrm{~km}^{2}\right)$. Quant au régime de type naturel, on observe une hausse et une baisse des valeurs de coefficients d'asymétrie pour quelques bassins versants. 


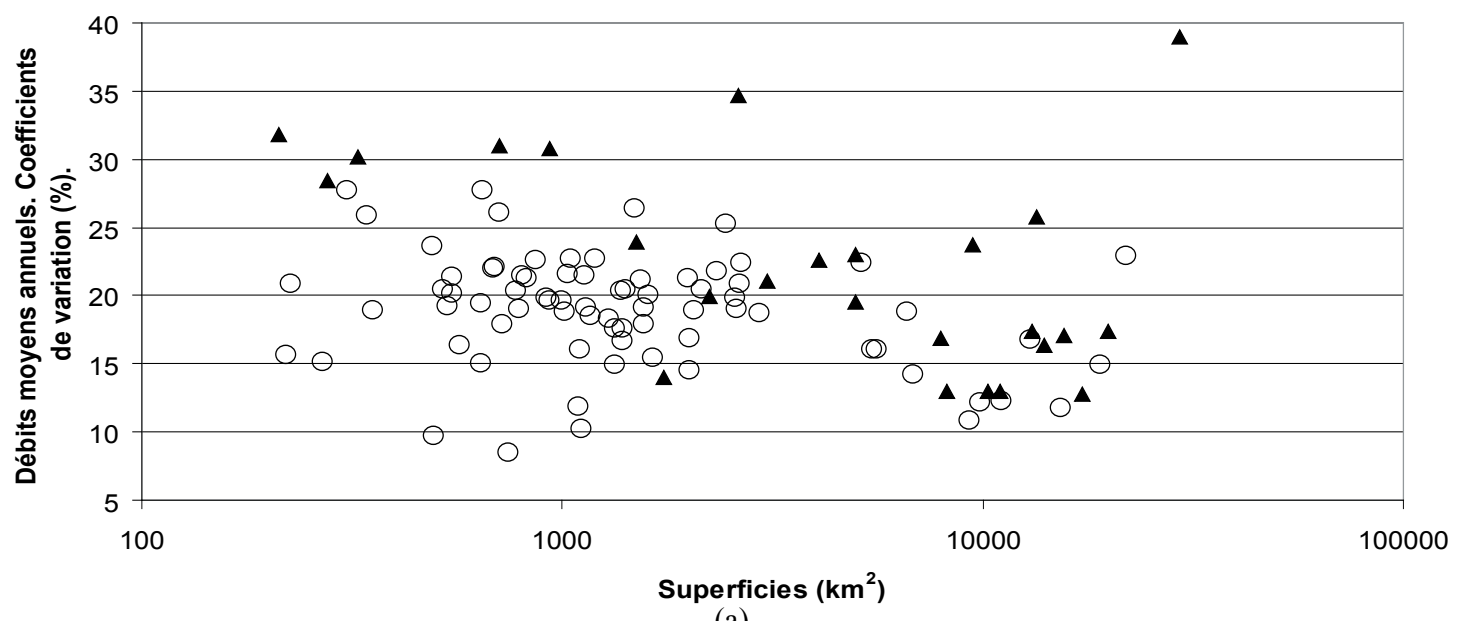

(a)

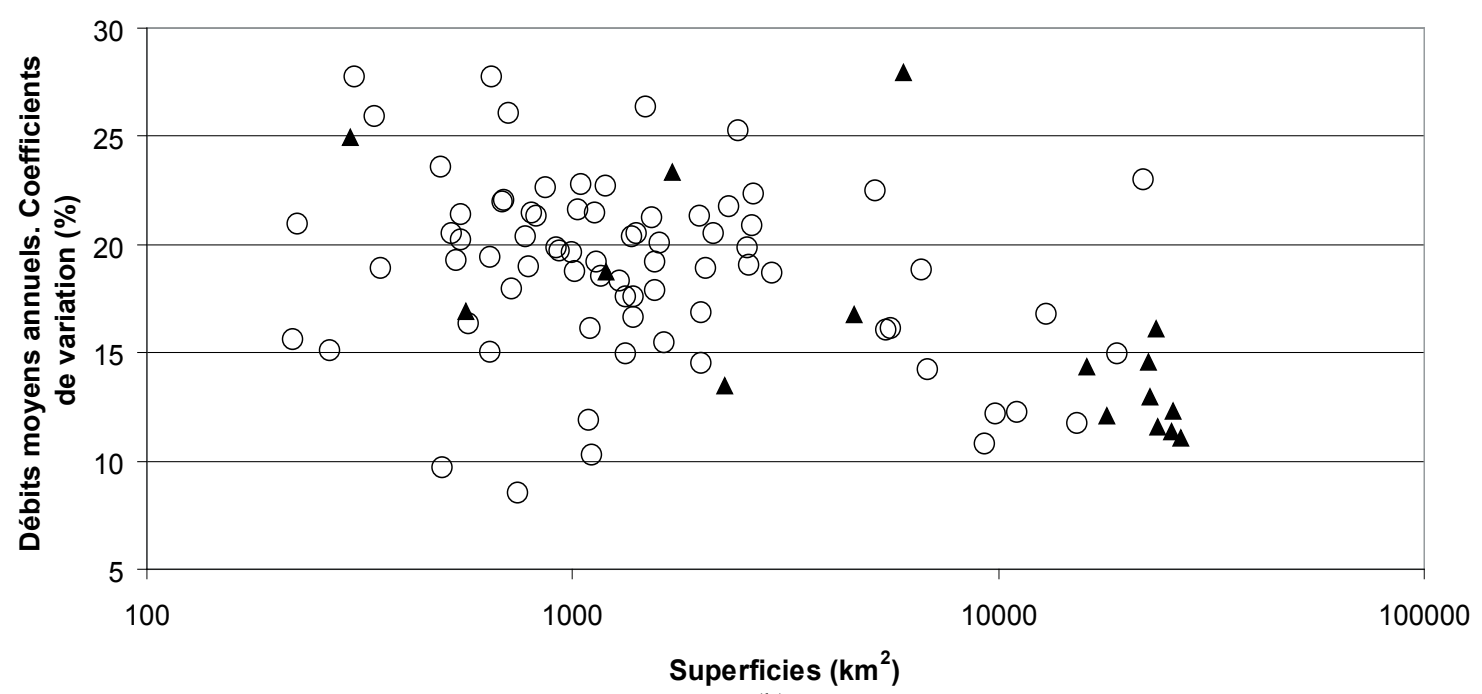

(b)

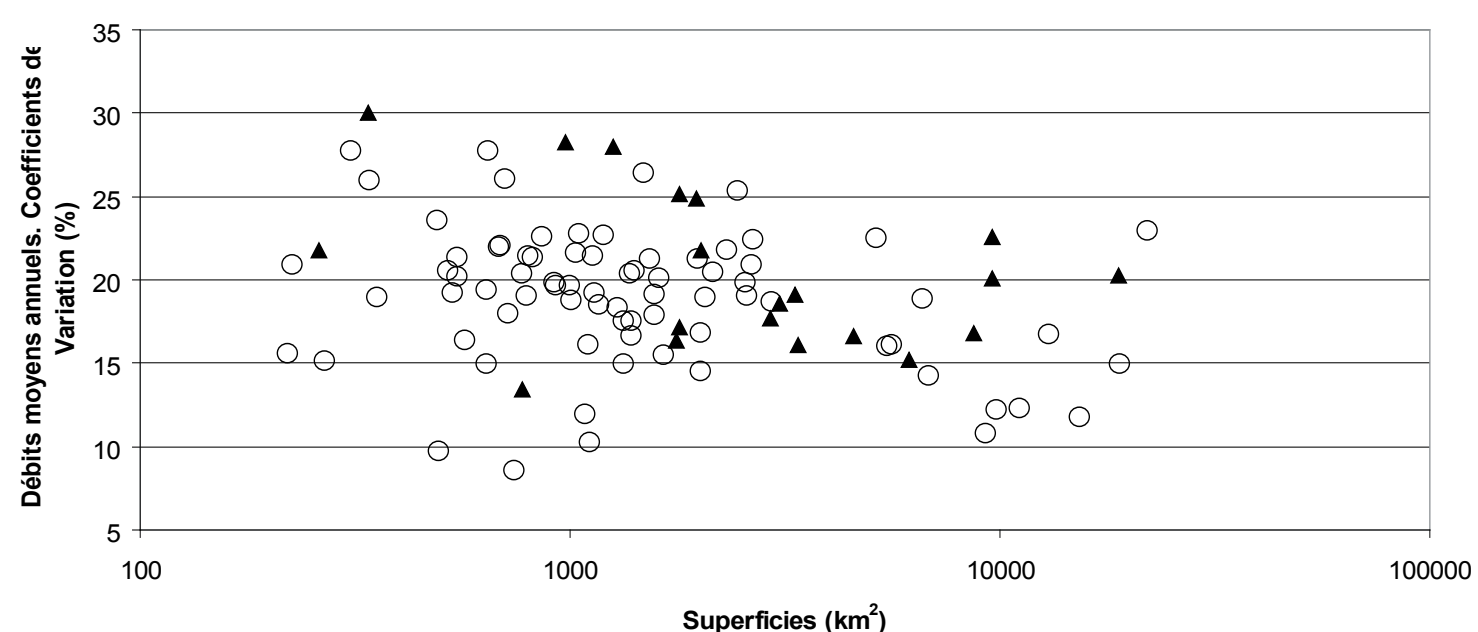

(c)

Figure 6. Relation entre superficies des bassins versants et coefficients de variation des débits moyens annuels en rivières naturelles (cercles) et régularisées (triangles); (a) régime d'inversion; (b) régime d'homogénéisation; (c) régime de type naturel.

Relationship between drainage area and coefficient of variation of MAF in unregulated (open circles) and regulated rives (triangles) (a) inversed regime; (b) homogenization regime; (c) natural type regime. 


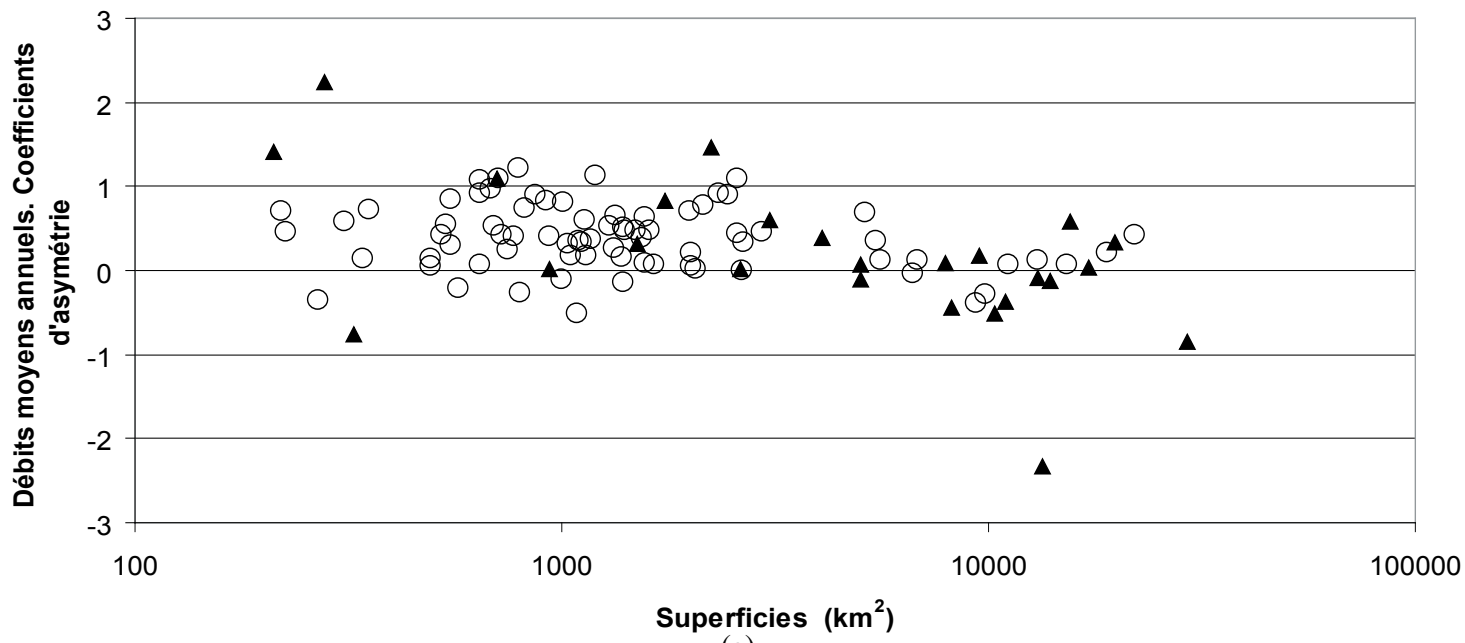

(a)

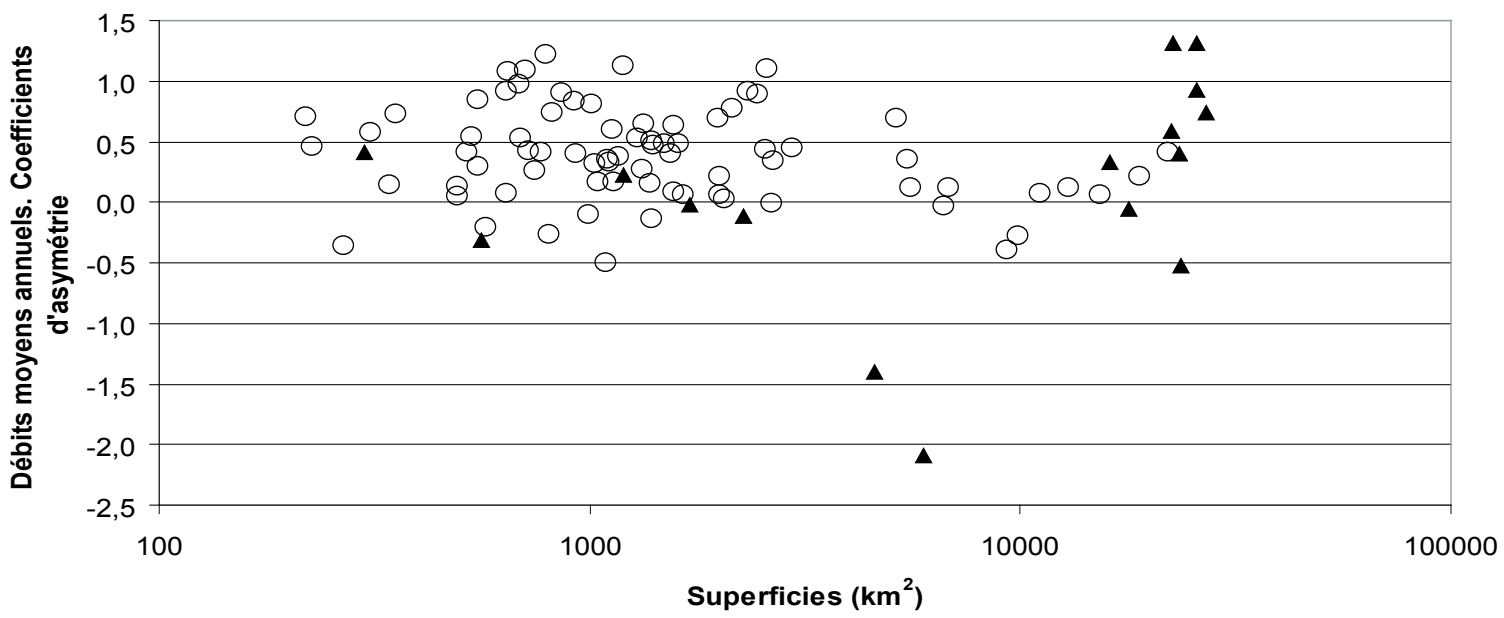

(b)

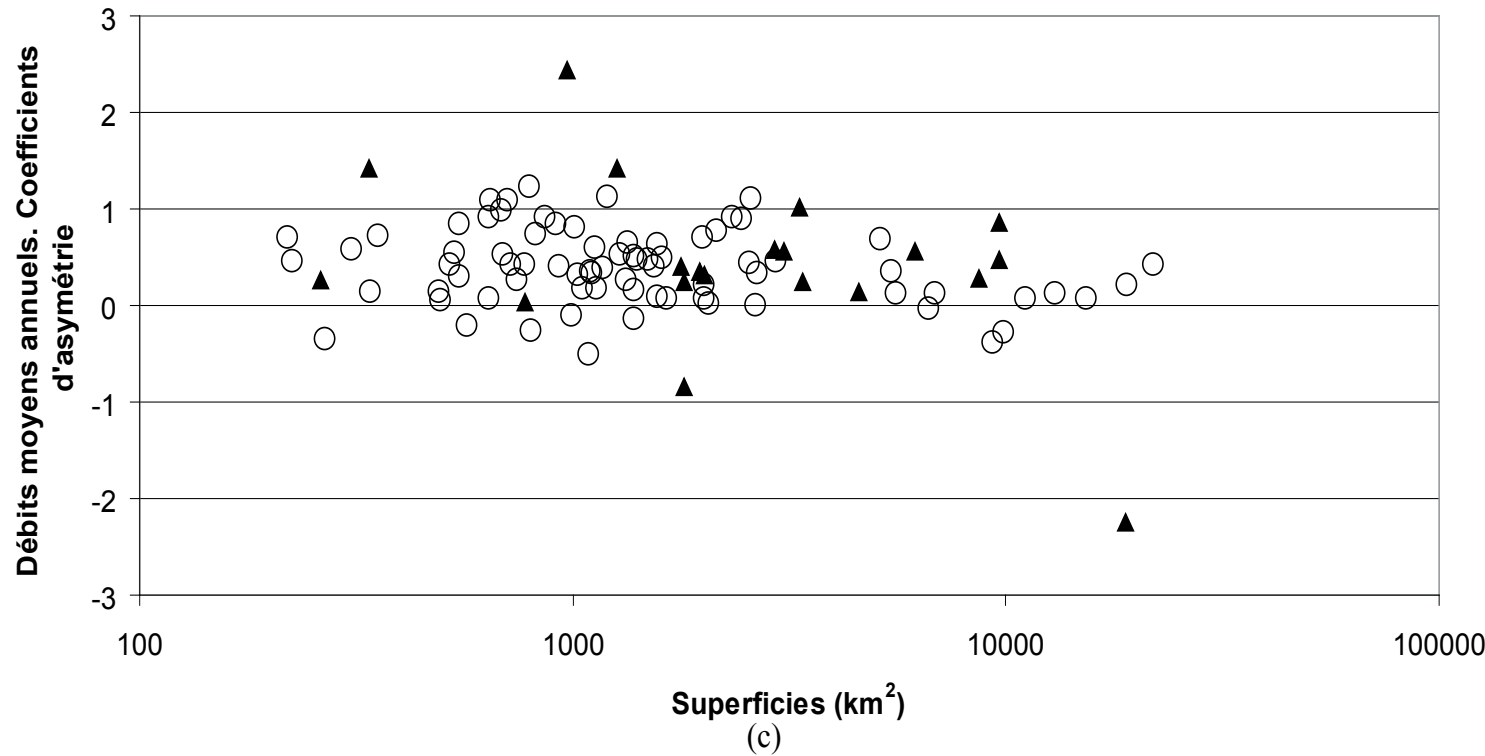

Figure 7. Relation entre superficies des basins versants et coefficients d'asymétrie des débits moyens annuels en rivières naturelles (cercles) et régularisées (triangles); (a) régimed'inversion; (b) régimed'homogénéisation; (c) régime de type naturel.

Relationship between drainage area and coefficients of skewness in unregulated (open circles) and regulated rivers (triangles); (a) inversed regime; (b) homogenization regime; (c) natural type regime. 


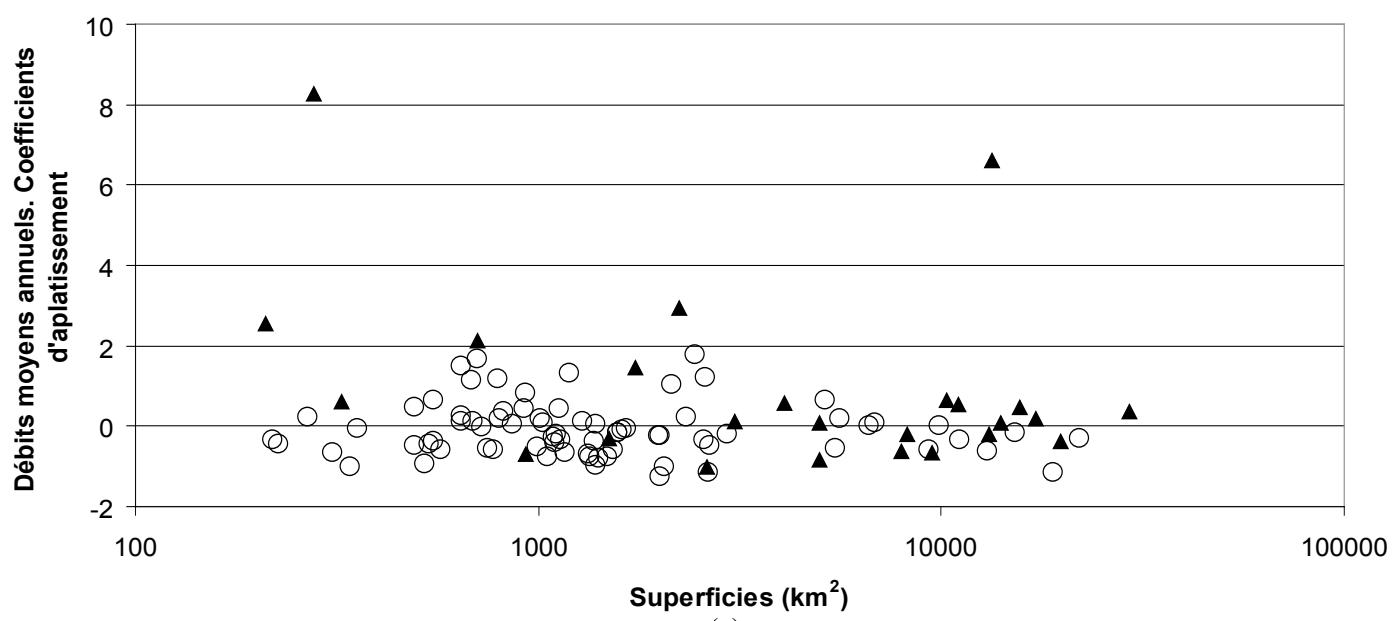

(a)

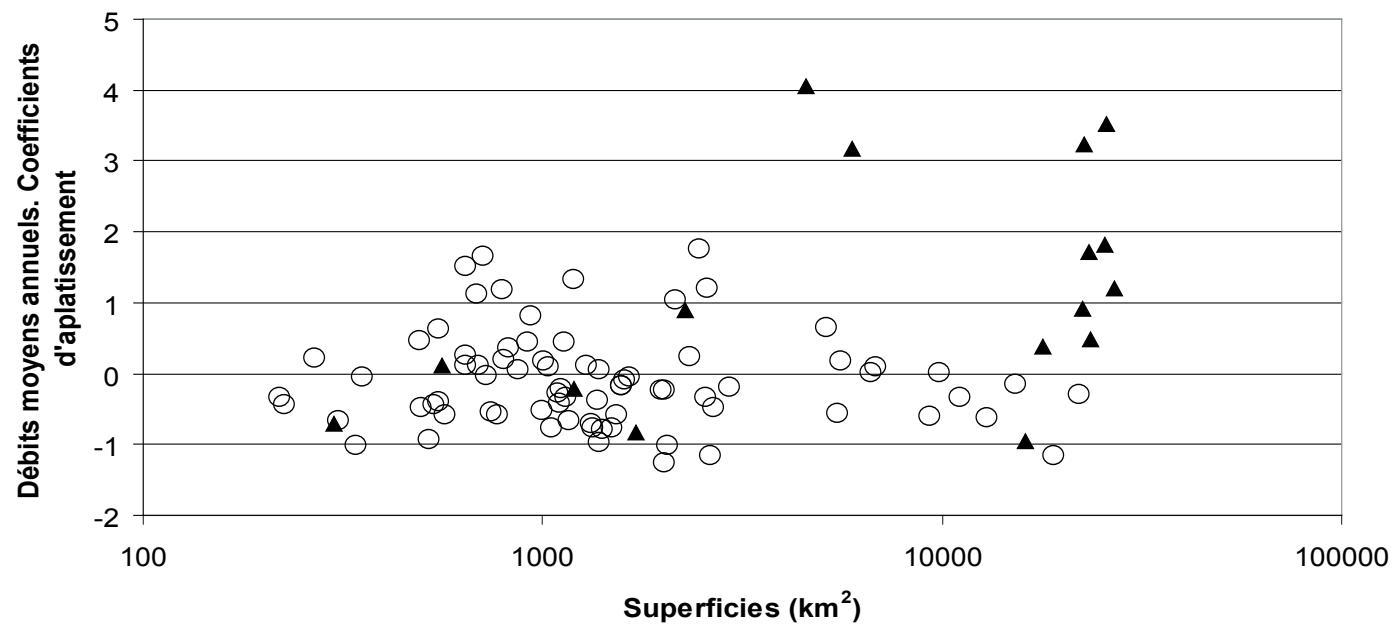

(b)

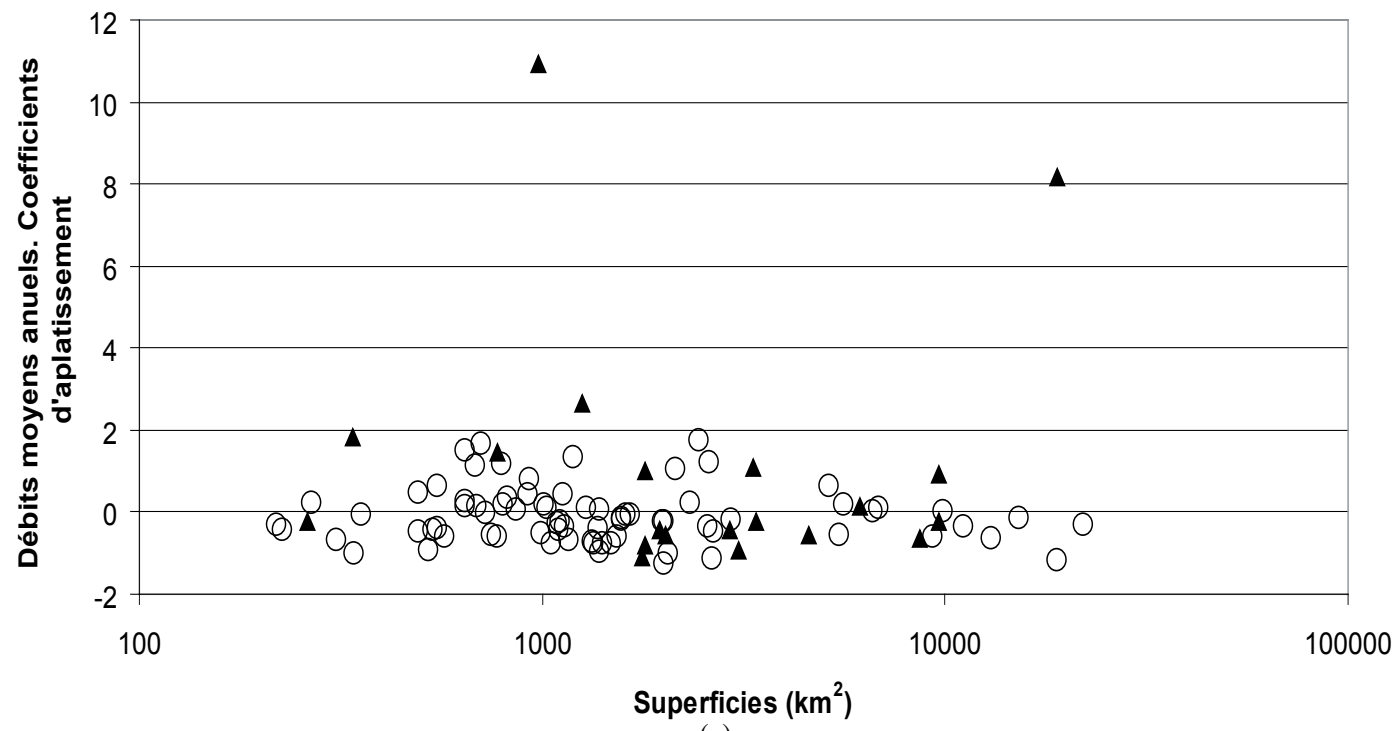

(c)

Figure 8. Relation entre superficies des bassins versants et coefficients d'aplatissement des débits moyens annuels en rivières naturelles (cercles) et régularisées (triangles) (a) régime d'inversion; (b) régime d'homogénéisation; (c) régime de type naturel.

Relationship between drainage area and coefficients of kurtosis in unregulated (open circles) and regulated rivers (triangles); (a) inversed regime; (b) homogenization regime; (c) natural type regime. 
Quant au coefficient d'aplatissement, on note une hausse des valeurs pour quelques rivières en aval des barrages (Figure 8). En effet, en rivières naturelles, la valeur du coefficient d'aplatissement n'excède pas 2. Ce seuil est fréquemment dépassé en aval des barrages. Le changement a été surtout observé en régime d'homogénéisation pour les rivières de taille supérieure à $5000 \mathrm{~km}^{2}$ (Figure $8 \mathrm{~b}$ ).

Ces résultats révèlent que les impacts de barrages sur la forme de distribution affectent surtout le régime d'homogénéisation et plus particulièrement les bassins versants de taille supérieure à $5000 \mathrm{~km}^{2}$. Ces impacts se traduisent par une hausse des valeurs de coefficients d'asymétrie et d'aplatissement.

\section{DISCUSSION}

L'analyse des impacts des barrages sur les débits de rivières poursuit quatre objectifs :

- La quantification des changements induits par les barrages sur les caractéristiques des débits. Il s'agit en fait de déterminer l'ampleur des modifications hydrologiques provoquées par un barrage. Jusqu'à présent, c'est le seul objectif poursuivi par toutes les études consacrées aux impacts hydrologiques des barrages. Mais cet objectif ne peut déboucher sur l'élaboration des politiques de gestion, de conservation et de restauration des tronçons régularisés. C'est la faiblesse majeure de cet objectif. Cette élaboration est la finalité de toute étude d'impacts hydrologiques induits par les barrages. Pour atteindre cette finalité, nous avons défini les objectifs supplémentaires suivants.

- La détermination des facteurs qui influencent l'ampleur des modifications hydrologiques induites par les barrages.

- La détermination des caractéristiques hydrologiques les plus modifiées par les barrages.

- Enfin, l'influence des conditions hydrologiques sur l'ampleur des modifications hydrologiques induites par les barrages.

\subsection{Méthodes de quantification de l'ampleur des impacts hydrologiques induits par les barrages}

En ce qui concerne la quantification de l'ampleur des modifications hydrologiques induites par les barrages, toutes les méthodes (méthodes de station témoin, de contrôle et de reconstitution) couramment utilisées dans la littérature sont destinées à atteindre cet objectif. Cependant, deux de ces trois méthodes présentent des faiblesses qui réduisent leur précision dans la quantification de l'ampleur des modifications hydrologiques des débits induite par un barrage. Dans le cas des méthodes de reconstitution, les données des débits reconstitués en condition naturelle sont entachées des erreurs liées au modèle hydrologique utilisé et aux formules de conversion de la quantité du kilowatts produite en débits d'eau. Deux modèles hydrologiques différents ne peuvent pas fournir exactement les mêmes valeurs de débits. Quant aux formules de conversion, les erreurs sont dues à une série de variables comme la mesure des niveaux du réservoir, l'estimation des débits évacués et turbinés, etc. Ces différentes erreurs sont difficiles à quantifier pour déterminer si l'écart entre les débits lâchés en aval d'un barrage et les débits reconstitués peut être considéré comme statistiquement significatif. La méthode de station contrôle ne permet pas une quantification précise des changements qui affectent les débits. C'est une méthode qualitative qui permet de conclure si il y a eu hausse ou baisse des débits après la construction d'un barrage. Ainsi, comparée à ces deux méthodes, la méthode de proportionnalité utilisée dans ce travail est beaucoup plus précise. Sa précision peut atteindre celle de la méthode de "station témoin" car les deux méthodes permettent de conclure sur la base d'un test statistique rigoureux si les débits mesurés en aval des barrages sont significativement différents de ceux mesurés en condition naturelle. La méthode de proportionnalité nous a donc permis de quantifier la diminution des débits annuels moyens en aval de certains barrages caractérisés par un régime d'inversion en période de sécheresse. C'est ainsi que nous avons observé des réductions des débits moyens annuels, durant certaines années sèches, parfois supérieures à $50 \%$ en aval de certains réservoirs. Cette réduction est même supérieure à celle provoquée par les transferts inter-bassins d'eau au Québec. En effet, la dérivation des eaux des rivières Koksoak et Caniaspicau, par exemple, a provoqué une diminution des débits moyens annuels respectivement de $35 \%$ et $48 \%$ après leur détournement vers le bassin versant de La Grande. Des réductions de débits encore plus importantes ont affecté les rivières Opinaca (87\%) et Eastmain $(90 \%)$ après leur détournement vers le même bassin (HAYEUR, 2001). Il convient cependant de noter que, contrairement aux dérivations dont les effets restent permanents, la réduction des débits observés en aval des réservoirs est temporaire et ne dure qu'une année.

\subsection{Détermination des facteurs qui influencent l'ampleur des modifications hydrologiques en aval des barrages}

Les changements hydrologiques qui affectent les caractéristiques des débits en aval des barrages sont complexes en raison de l'interaction de nombreux facteurs. Cinq facteurs ont été répertoriés pour rendre compte de l'ampleur de ces changements (ASSANI et al., 2005, 2006; BATALLA et al., 2004; DYNESIUS et NILSSON, 1994) :

- Le mode de gestion des barrages. Il dépend des objectifs assignés à ceux-ci. Ainsi, par exemple, un barrage-réservoir ne provoque pas les mêmes modifications hydrologiques 
qu'une centrale hydroélectrique destinée simplement à produire l'énergie électrique. De même, un barrage destiné à produire seulement de l'électricité ne fonctionnera pas de la même façon qu'un barrage destiné à la fois à la production de l'énergie électrique et à l'irrigation ou encore un barrage destiné à fournir de l'eau à une agglomération.

- La taille du bassin versant. Elle détermine la dimension des barrages et/ou son mode de gestion. La gestion d'un réservoir construit sur un grand cours d'eau peut ou non provoquer les mêmes effets que celle d'un réservoir érigé sur un petit cours d'eau. La taille du bassin versant et le mode de gestion influencent la capacité des réservoirs, variable utilisée pour expliquer l'ampleur des changements hydrologiques en aval (BATALLA et al., 2004).

- Le nombre de barrages construit sur un même cours d'eau. On peut aisément concevoir que les impacts hydrologiques seront beaucoup moins importants sur une rivière avec un seul barrage que sur une rivière de même taille avec de nombreux barrages.

- Lâge des barrages. En raison de l'émergence de la conscience environnementale depuis les années 1970, les impacts hydrologiques des barrages tendent à être atténués. On peut ainsi concevoir que la gestion des barrages construits récemment est susceptible de causer moins d'impacts hydrologiques que celle des anciens barrages parce qu'on ne tenait pas compte des préoccupations environnementales lors de la construction des barrages avant 1970 au Québec (HAYEUR, 2001).

- Le climat. Le climat détermine le régime hydrologique des rivières, d'une part, et le taux d'évapotranspiration, d'autre part. Les impacts hydrologiques d'un barrage destiné à la production de l'énergie électrique en saison froide peuvent significativement être différents dans un climat tempéré océanique caractérisé par des hivers doux et pluvieux que dans un climat tempéré froid caractérisé par des hivers rigoureux et secs. De même, un réservoir en climat sec et chaud sera soumis à une plus forte évapotranspiration qu'un réservoir en climat tempéré et pluvieux.

Pour mettre en évidence l'influence de ces facteurs, il faut analyser simultanément toutes les stations, comme c'est le cas avec la méthode de proportionnalité utilisée dans ce travail. Ainsi, grâce à celle-ci, nous avons pu déterminer l'influence de deux facteurs sur les débits moyens annuels au Québec, à savoir le mode de gestion et la taille des bassins versants. En ce qui concerne le mode de gestion, nous avons démontré que les modifications hydrologiques affectent surtout les barrages caractérisés par le régime d'inversion. Ces modifications se traduisent par une baisse du volume d'écoulement pendant les années hydrologiques sèches et une forte variabilité interannuelle des débits ainsi que, dans une moindre mesure, par une baisse des valeurs des coefficients d'asymétrie et une hausse des valeurs des coefficients d'aplatissement. Quant au régime d'homogénéisation, les modifications induites par les barrages affectent seulement les coefficients d'asymétrie et d'aplatissement. Enfin, le régime de type naturel est le moins modifié par les barrages. Ces résultats confirment ceux déjà observés aux échelles mensuelles et journalières (Tableau 3). Ainsi, les modifications hydrologiques induites par les barrages affectent surtout le régime d'inversion à toutes les échelles. Rappelons que ce régime est exclusivement associé aux réservoirs (ASSANI et al., 2004). Ceux-ci sont destinés généralement à alimenter en eau les centrales hydroélectriques situées en aval pendant la période hivernale et pour prévenir les inondations au printemps au moment de la fonte des neiges. Ainsi, au printemps (avril et mai), on stocke l'eau provenant de la fonte des neiges pour reconstituer les réserves d'eau qui seront utilisées en hiver, saison durant laquelle l'écoulement devient faible en raison du stockage des eaux de précipitations sous forme de neige. Le régime de type inversion est un régime rarement observé dans le monde. Mais, au Québec, il représente plus du tiers des barrages hydroélectriques en raison du climat particulièrement

Tableau 3. Comparaison des impacts des barrages en fonction de leurs modes de gestion aux échelles annuelles, mensuelles et journalières au Québec.

Table 3. Comparison of dams impacts according to the management mode at annual, monthly and daily scales.

\begin{tabular}{|c|c|c|c|c|c|c|c|c|c|}
\hline \multirow[t]{2}{*}{ Caractéristiques } & \multicolumn{3}{|c|}{ Échelle annuelle } & \multicolumn{3}{|c|}{ Échelle mensuelle* } & \multicolumn{3}{|c|}{ Échelle journalière** } \\
\hline & RI & RH & RTN & RI & RH & RTN & RI & $\mathrm{RH}$ & RTN \\
\hline VE-Fréquence & $\mathrm{X}$ & & & $\mathrm{X}$ & $\mathrm{X}$ & & $\mathrm{X}$ & $\mathrm{X}$ & $\mathrm{X}$ \\
\hline Variabilité du VE & $\mathrm{X}$ & & & $\mathrm{X}$ & & & $\mathrm{X}$ & $\mathrm{X}$ & \\
\hline PO & & & & $\mathrm{X}$ & $\mathrm{X}$ & & $\mathrm{X}$ & $\mathrm{X}$ & \\
\hline Variabilité de la PO & & & & $\mathrm{X}$ & $\mathrm{X}$ & & $\mathrm{X}$ & & \\
\hline $\mathrm{C}_{\mathrm{s}}$ & $\mathrm{X}$ & $\mathrm{X}$ & $\mathrm{X}$ & $\mathrm{X}$ & $\mathrm{X}$ & & $\mathrm{X}$ & $\mathrm{X}$ & \\
\hline$C_{k}$ & $\mathrm{X}$ & $\mathrm{X}$ & $\mathrm{X}$ & $\mathrm{X}$ & $\mathrm{X}$ & & $\mathrm{X}$ & $\mathrm{X}$ & \\
\hline
\end{tabular}

$\mathrm{RI}$ = régime d'inversion; $\mathrm{RH}=$ Régime d'homogénéisation; $\mathrm{RTN}$ = régime de type naturel; $\mathrm{VE}=$ volume d'écoulement; $\mathrm{PO}=$ période d'occurrence des débits maximums et minimums; $\mathrm{C}_{\mathrm{s}}=$ coefficient d'asymétrie; $\mathrm{C}_{\mathrm{k}}=$ coefficient d'aplatissement; $\mathrm{X}=$ changement de la caractéristique des débits. * : source : LAJOIE et al., 2006 ; ** : source : ASSANI et al. (2005, 2006). 
froid en hiver. Mais, lorsque la taille des bassins versants devient importante pour soutenir l'écoulement hivernal, la quantité d'eau stockée dans les réservoirs au printemps diminue. On passe ainsi au régime dit "d'homogénéisation». Celui-ci se distinguefondamentalement du régime d'inversion parl'absence d'occurrence des débits minimums mensuels au printemps au moment de la fonte des neiges. Le régime d'homogénéisation est le régime le plus couramment observé en climat tempéré continental ou de montagne en raison d'un hiver relativement froid nécessitant une forte production d'énergie électrique pour le chauffage (ANDREWS et PIZZI, 2000; ERSKINE et al., 1999; LECONTE et al., 2001; MAHESHWARI et al., 1995; MERRIT et COOPER, 2000; REILY et JOHNSON, 1982). L'eau stockée au printemps peut servir aussi à l'irrigation ou à la climatisation en été. Quant au régime du type naturel, il est associé le plus souvent aux barrages construits au fil de l'eau ou aux centrales hydroélectriques pour produire de l'énergie. La plupart de ces barrages ne disposent donc pas de réservoirs. En hiver, ils sont alimentés directement par les réservoirs situés généralement en amont (régime d'inversion). Au printemps, au moment du stockage des eaux dans les réservoirs, ils sont directement alimentés par les eaux de fonte de neige apportées par les affluents non régularisés. Pendant cette période, la production de l'énergie baisse en raison du réchauffement de la température. Toutefois, certains barrages de cette catégorie servent à lutter contre les inondations. Ils peuvent ainsi stocker assez d'eau au printemps, sans pour autant entraîner une inversion du cycle naturel des débits.

L'ampleur des modifications hydrologiques induites par les barrages dépend aussi de la taille des bassins versants. À l'échelle annuelle, cette influence de la taille des bassins versants a été surtout manifeste en régime d'homogénéisation. Ainsi, nous avons observé une hausse des coefficients d'asymétrie pour les rivières de taille supérieure à $10000 \mathrm{~km}^{2}$ mais une baisse pour les bassins versants de taille comprise entre 5000 et $10000 \mathrm{~km}^{2}$.
Quant aux rivières de taille inférieure à $5000 \mathrm{~km}^{2}$, aucun changement n'a été observé.

Enfin, la méthode de proportionnalité ne permet pas de tenir compte simultanément de tous les cinq facteurs qui influencent l'ampleur des changements hydrologiques en aval des barrages. Nous comptons ainsi utiliser plus tard des méthodes statistiques multivariées et complexes comme l'analyse des corrélations canoniques (méthode linéaire) ou l'analyse neuronale (méthode non linéaire) pour tenir compte de tous ces facteurs. Mais contrairement à la méthode de proportionnalité, ces dernières méthodes ne permettent pas de comparer les débits en rivières naturelles et en rivières régularisées, point commun à toutes les méthodes d'étude d'impacts de barrages, ce qui pose un problème théorique dans leur application.

\subsection{Détermination des caractéristiques des débits les plus modifiées en aval des barrages}

Toutes les variables hydrologiques des débits ne sont pas modifiées de la même façon par les barrages. Cet aspect est peu étudié. Ainsi, beaucoup de travaux portant sur les débits moyens annuels se limitent souvent à l'analyse d'une seule caractéristique, à savoir la moyenne (volume d'écoulement) des débits moyens annuels (CHURCH, 1995; LOIZEAU et DOMINIK, 2000; VIVIAN, 1994; WEINGARTNER et ASCHWANDEN, 1994). Comme l'avaient notamment déjà souligné RICHTER et al. (1996), cette approche est insuffisante sur le plan écologique du fait qu'elle ne peut rendre compte de toutes les modifications écologiques induites par un barrage. En effet, selon le concept de « régime des débits naturels ", chaque caractéristique des débits joue un rôle précis dans le fonctionnement d'un écosystème aquatique (Tableau 4). Toute modification qui affecte une seule de ces caractéristiques peut entraîner des changements plus ou moins

Tableau 4. Les cinq caractéristiques fondamentales et leurs rôles écologiques (RICHTER et al., 1996).

Table 4. The five flow characteristics and their ecological roles (RICHTER et al., 1996).

\begin{tabular}{ll}
\hline Caractéristiques & Exemples des rôles écologiques \\
\hline Magnitude & $\begin{array}{l}\text { Mesure du volume d'habitats disponible pour les espèces aquatiques ou semi-aquatiques. } \\
\text { Mesure de la position de la nappe par rapport aux systèmes racinaires de la végétation } \\
\text { riparienne. }\end{array}$ \\
Peut déterminer si certains cycles vitaux seront satisfaits ou non et peut influencer le degré \\
de stress associé aux conditions extrêmes telles les inondations ou les sécheresses. \\
Fréquence & $\begin{array}{l}\text { Peut être reliée à la reproduction et à la mortalité des espèces, influençant ainsi la dynamique } \\
\text { des populations. }\end{array}$ \\
Purée & $\begin{array}{l}\text { Peut déterminer si des phases particulières des cycles vitaux peuvent être complétées } \\
\text { ounout affecter le degré de stress associé aux évènements extrêmes comme les }\end{array}$ \\
Variabilité & $\begin{array}{l}\text { Indique la variabilité du volume d’habitats disponibles et peut influencer la capacité de la } \\
\text { végétation à maintenir un contact avec la nappe phréatique. }\end{array}$ \\
\hline
\end{tabular}


profonds de ce fonctionnement. Il est donc impératif de tenir compte de toutes les caractéristiques de débits dans les études d'impacts hydrologiques de barrages. Ceci se justifie par le fait que certaines caractéristiques peuvent être plus modifiées que d'autres par les barrages. L'identification des caractéristiques modifiées permet d'apporter des solutions concrètes et précises pour restaurer les tronçons régularisés.

Le changement le plus important mis en évidence dans ce travail est sans nul doute la diminution du volume d'écoulement des débits moyens annuels pendant les années sèches et la forte variabilité interannuelle des débits en régime d'inversion. L'analyse détaillée des débits en amont et en aval du réservoir Taureau construit sur la rivière Matawin, caractérisé par un régime d'inversion, ainsi que les observations de terrain effectuées entre 2002 et 2005 sur la même rivière, permettent d'expliquer aisément cette baisse et cette variabilité (ASSANI et al., 2002). En effet, durant les années sèches, on stocke davantage d'eau de fonte dans le réservoir afin d'éviter toute pénurie en hiver pour la production de l'énergie électrique. Ainsi, au printemps et en été, on lâche très peu d'eau en aval du réservoir provoquant une diminution significative des débits printaniers et estivaux. Au mois de mai par exemple, les débits moyens mensuels minimums en aval des barrages sont beaucoup plus faibles qu'en rivières naturelles (Figure 9). C'est le cas observé en 2003, qui fut une année particulièrement sèche, en aval du réservoir Taureau. Durant cette année, le débit était quasi nul entre avril et juillet au point que le lit de la rivière était presque à sec à de nombreux endroits permettant même à la végétation d'envahir le lit mineur (GRAVEL, 2005). En revanche, durant les années hydrologiques humides et normales, le stockage de l'eau dans les réservoirs est limité. Ainsi, les impacts sur les débits moyens annuels sont fortement atténués malgré l'écrêtement des crues observé en hiver. C'est le cas de l'année 2004 qui fut plus humide que la normale. Le niveau d'eau en aval du réservoir Taureau était comparable à celui des rivières naturelles entre avril et juillet. Si le mode de gestion est la principale cause de la baisse des débits moyens annuels en période de sécheresse, on ne peut négliger l'influence des conditions climatiques. En effet, les années sèches sont caractérisées par de longues périodes d'ensoleillement, des températures de l'air relativement élevées et un faible taux d'humidité de l'air. Ces conditions climatiques peuvent donc favoriser une forte évaporation de l'eau stockée dans les réservoirs au printemps et en été contribuant ainsi à une diminution des débits moyens annuels pendant les années hydrologiques sèches.

\section{CONCLUSION}

Contrairement à l'opinion couramment admise, les barrages peuvent modifier les caractéristiques des débits moyens annuels au Québec. Mais l'ampleur de cette modification hydrologique dépend du mode de gestion des barrages, de la taille des bassins versants et de la variable hydrologique analysée. En ce qui concerne le mode de gestion, ce sont les régimes d'inversion et d'homogénéisation qui sont affectés. Quant à la taille des bassins versant, son influence a été observée seulement en régime d'homogénéisation. Les changements hydrologiques

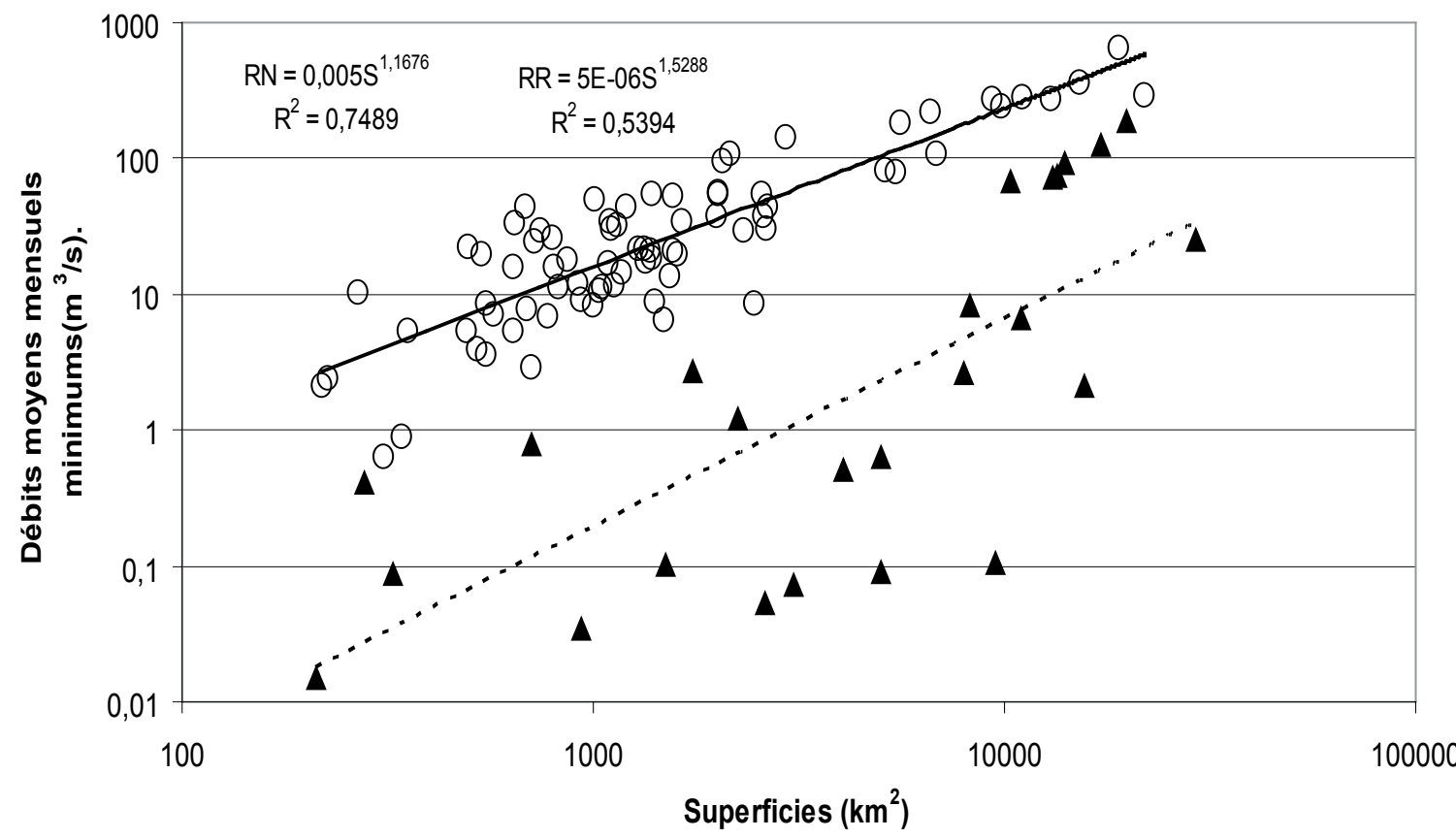

Figure 9. Comparaison de la relation entre superficies des bassins versants et débits moyens mensuels minimums en rivières naturelles (cercles et ligne continue, $\mathrm{RN}$ ) et régularisées (triangles et ligne discontinue, $\mathrm{RRI}$ ) au mois de mai en régime d'inversion.

Comparison of the relationship between drainage area and minimum monthly flows in unregulated rivers (open circles and unbroken line, $R N$ ) and inversely regulated rivers (triangles and broken line, $R N$ ) in May. 
ont surtout affecté les bassins versants de taille supérieure à $5000 \mathrm{~km}^{2}$. Enfin, en régime d'inversion, les impacts des barrages se traduisent par une baisse des débits moyens annuels pendant les années sèches et une variabilité inter-annuelle des débits relativement plus forte que celle des rivières naturelles. En régime d'homogénéisation, ces impacts se traduisent par une hausse des valeurs de coefficients d'asymétrie et d'aplatissement. Ces résultats révèlent que le type de caractéristique hydrologique modifiée par les barrages peut varier d'un régime hydrologique à un autre : le volume d'écoulement-fréquence en régime d'inversion et la forme de la courbe de distribution (coefficients d'asymétrie et d'aplatissement) en régime d'homogénéisation.

Ces trois facteurs (mode de gestion des barrages, taille des bassins versants et variable hydrologique analysée) doivent être pris en compte dans l'élaboration des mesures de restauration et de conservation des écosystèmes de tronçons régularisés au Québec parce que leur influence a été mise en évidence à toutes les échelles temporelles (année, saison, mois, jour) dans le cadre de nos travaux (ASSANI, 2004; ASSANI et al., 2005, 2006, LAJOIE et al, 2007).

\section{RÉFÉRENCES BIBLIOGRAPHIQUES}

ANCTIL F. et P. COULIBALY (2004). Wavelet analysis of the interannual variability in Southern Québec streamflow. J. Clim., 17, 163-173.

ANDREWS E.D. et A.L. PIZZI (2000). Origin of Colorado River experimental flood in Grand Canyon. Hydrol. Sci. J., 45, 607-627.

ASSANI A.A. (2003). Nouvelle théorie de régionalisation des débits : la théorie éco-géographique. Notes de recherche No 01/03, Laboratoire d'hydro-climatologie et de géomorphologie fluviale, UQTR, 10p.

ASSANI A.A. (2004). Comparaison de la variabilité interannuelle des débits en fonction de régimes hydrologiques artificialisés dans un complexe de barrages sur la rivière Saint-Maurice (Québec, Canada). Bull. Soc. Geogr. Liège, 44, 87-96.

ASSANI A.A., T. BUFFIN-BÉLANGER et A.G. ROY (2002). Analyse d'impacts d'un barrage sur le régime hydrologique de la rivière Matawin (Québec, Canada). Rev. Sci. Eau, 15, 557-574.

ASSANI A.A., E. GRAVEL, T. BUFFIN-BÉLANGER et A.G. ROY (2005). Impacts des barrages sur les débits minimums annuels en fonction des régimes hydrologiques artificialisés. Rev. Sci. Eau, 18, 103-127.

ASSANI A.A., F. PETIT et G. MABILLE (1999). Analyse des débits de la Warche aux barrages de Butgenbach et de Robertville (Ardenne belge). Bull. Soc. Geogr. Liège, 36, 17-30.

ASSANI A.A., E. STICHELBOUT, A.G. ROY et F. PETIT (2006). Comparison of impacts of dams on the annual maximum flow characteristics in the three regulated hydrologic regimes in Québec. Hydrol. Process., 20, 3485-3501.

BATALLA R.M., C.M. GOMEZ et G.M. KONDOLF (2004). Reservoir-induced hydrological changes in the Ebro River basin (NE Spain). J. Hydrol., 290, 117-136.

BELZILE L., P. BÉRUBÉ, V.D. HOANG et M. LECLERC (1997). Méthode écohydrologique de détermination des débits réservés pour la protection des habitats du poisson dans les rivières du Québec. Rapport soumis par l'INRS-Eau et Groupe-conseil Génivar inc. au ministère de l'Environnement et de la Faune et Pêches et Océans Canada, Rapport $\mathrm{N}^{\circ} \mathrm{R}-494,83$ p. +8 annexes.

BENN P.C. et W.D. ERSKINE (1994). Complex channel response to flow regulation: Cudgegong River below Windamere Dam, Australia. Appl. Geogr., 14, 153-168.

BIGGS B.J.F., N.C. TUCHMAN, R.L. LOWE et R.J. STEVENSON (1999). Resource stress alters hydrological disturbance effects in a stream periphyton community. Oiko, 85, 95-108.

BIGGS B.J.F., I.N. VLADIMIR et T.H. SNELDER (2005). Linking scales of flow variability to lotic ecosystems structure and function. River Res. Appl., 21, 283-298.

CHURCH M. (1995). Geomorphic response to river flow regulation: case studies and time-scales. Regul. Rivers: Res. Manage., 11, 3-22.

DYNESIUS M. et C. NILSSON (1994). Fragmentation and flow regulation of rivers systems in the northern third of the world. Sci., 266, 753-762.

ENVIRONNEMENTCANADA (1996). HYDAT CDROM : Données sur les eaux de surface et sur les sédiments jusqu'en 1994. CDROM 2245.

ERSKINE W.D., N. TERRAZZOLO et R.F. WARNER (1999). River rehabilitation from the hydrogeomorphic 
impacts of large hydro-electric power project: Snowy River, Australia. Regul. Rivers: Res. Manage., 15, 3-24.

GRAVEL É. (2005). Effets de la hausse des débits sur les paramètres physico-chimiques de l'eau et des sédiments ainsi que sur la richesse spécifique de la végétation du lit mineur en aval du réservoir Taureau sur la rivière Matawin (Québec). Congrès annuel de l'ACG, London (Ontario), juin 2005 (résumé).

HAYES J.W. (1995). Spatial and temporal variation in the relative density and size of juvenile brown trout in the Kakanui River, North Otago, New Zealand. New Zealand J. Mar. Freshwater Res., 29, 393-408.

HAYEUR G. (2001). Synthèse des connaissances environnementales acquises en milieu nordique de 1970 à 2000. Hydro-Québec, Montréal, 110 p.

HUDON C. (1997). Impact of water level fluctuation on St. Lawrence river aquatic vegetation. Can. J. Fish. Aquat. Sci., 54, 2853-2865.

HUDON C. (2004). Shift in wetland plant composition and biomass following low-level episodes in the St. Lawrence river: looking into the future. Can. J. Fish.Aquat. Sci., 61, 603-617.

LAJOIE F., A.A. ASSANI, A.G. ROY et M. MESFIOUI (2007). Impacts of dams on monthly flow characteristics. The influence of watershed size and seasons. J. Hydrol., 334, 423-439.

LECONTE R., A. PIETRONIRO, D.L. PETERS et T.D. PROWSE (2001). Effects of flow regulation on hydrologic patterns of large, inland delta. Regul. Rivers: Res. Manage., $17,51-65$.

LOIZEAU J.L. et J. DOMINIK (2000). Evolution of the Upper Rhone River discharge and suspended sediment load during the last 80 years and some implications for lake Geneva. Aquat. Sci., 62, 54-67.

MAGILLIGAN F.J. et K.H. NISLOW (2005). Changes in hydrologic regime by dams. Geomorph., 71, 61-78.

MAHESHWARI B.L., K.F. WALKER et A. MCMAHON (1995). Effects of regulation on the flow regime of the River Murray, Australia. Regul. Rivers: Res. Manage., 10, 15-38.

McINTOCH A.R. (2000). Habitat- and size-related variations in exotic trout impacts on native galaxid fishes in New Zealand. Can. J. Fish. Aquat. Sci., 57, 2140-2151.
MERRITTD.M.et D.J. COOPER (2000). Riparian vegetation and channel change in response to river regulation: a comparative study of regulated and unregulated streams in the Green River basin, USA. Regul. Rivers: Res. Manage., $16,543-564$.

PETERS D.L. et T. PROWSE (2001). Regulation effects on the lower Peace River, Canada. Hydrol. Process., 15, 3181-3194.

POFF N.L, J.D. ALLAN, M.B. BAIN, J.G. KARR, K.L. PRESTEGAARD, B.D. RICHTER, R.E. SPARKS et J.C. STROMBERG (1997). The natural flow regime. A paradigm for river conservation and restoration. BioSci., 47, 769-784.

REILY P.W. et W.C. JOHNSON (1982). The effects of altered hydrologic regime on tree growth along the Missouri River in North Dakota. Can. J. Bot., 60, 2410-2423.

RITCHER B.D., J.V. BAUMGARTNER, J. POWELL et D.P. BRAUN (1996). A method for assessing hydrologic alteration within ecosystem. Conserv. Biol., 10, 1163-1174.

VIVIAN H. (1994). L'hydrologie artificialisée de l'Isère en amont de Grenoble. Essai de quantification des impacts des aménagements. Rev. Geogr. Alpine, 2, 97-112.

WEINGARTNER R. et H. ASCHWANDEN (1994). Quantification des débits des cours d'eau des Alpes suisses et des influences anthropiques qui les affectent. Rev. Geogr. Alpine, 2, 45-58. 